S. Kaneyuki and H. Asano

Nagoya Math. J.

Vol. 112 (1988), 81-115

\title{
GRADED LIE ALGEBRAS AND GENERALIZED JORDAN TRIPLE SYSTEMS
}

\author{
SOJI KANEYUKI AND HIROSHI ASANO \\ Dedicated to Professor Akihiko Morimoto on his sixtieth birthday
}

\section{Introduction}

One frequently encounters (real) semisimple graded Lie algebras in various branches of differential geometry (e.g. [16], [9], [14], [18]). It is therefore desirable to study semisimple graded Lie algebras, including those which have been studied individually, in a unified way. One of our concerns is to classify (finite-dimensional) semisimple graded Lie algebras in a way that enables us to construct them. A graded Lie algebra $\mathfrak{g}$ of the form $\mathfrak{g}=\sum_{k=-\nu}^{\nu} \mathfrak{g}_{k}$ is said to be of the $\nu$-th kind. The classification of semisimple graded Lie algebras of the $\nu$-th kind was done by KobayashiNagano [4] for $\nu=1$, and by J.H. Cheng [3] for $\nu=2$ and $\operatorname{dim} g_{-2}=1$. The first aim of this paper is to obtain a classification theorem (Theorem 1.7) for semisimple graded Lie algebras, which establishes a bijective correspondence between isomorphism classes of all gradations in a real semisimple Lie algebra $\mathfrak{g}$ and certain equivalence classes of partitions $\left(\Pi_{0}, \Pi_{1}, \cdots, \Pi_{s}\right)$ of a restricted fundamental root system $\Pi$ of g. For the complex semisimple case, a similar but weaker assertion has been obtained by V.G. Kac [5]. Theorem 1.7 and its proof enable us to construct all gradations in a semisimple Lie algebra. A graded Lie algebra $\mathfrak{g}=\sum_{k=-\infty}^{\infty} \mathfrak{g}_{k}$ (not necessarily of finite dimension) is said to be of type $\alpha_{0}$, if $\sum_{k \leqslant-1} \mathfrak{g}_{k}$ and $\sum_{k \geqslant 1} \mathfrak{g}_{k}$ are generated by $\mathfrak{g}_{-1}$ and $\mathfrak{g}_{1}$ respectively. In Theorem 2.6 we give a necessary and sufficient condition for a gradation to be of type $\alpha_{0}$. By using this, we will construct explicitly (up to isomorphisms) all gradations of the first and the second kind in each classical real simple Lie algebra ( $\S \S 2.3$ and 4.2 ).

Our second concern is the problem of classifying a wider class of triple systems, called generalized Jordan triple systems which contain all

Received April 22, 1987. 
Jordan triple systems as a special case. To this problem we apply the classification and construction of semisimple graded Lie algebras given in $\S \S 1$ and 2. To a Jordan triple system there associates a graded Lie algebra of the first kind [9]. I.L. Kantor [7] considered a similar situation in a more general setting to obtain: To a generalized Jordan triple system $\left(U_{-1}, B\right)$ there corresponds a graded Lie algebra $\mathscr{L}(B)$ which is not necessarily of finite dimension. If the graded Lie algebra $\mathscr{L}(B)$ is of the $\nu$-th kind (resp. classical), then we say that $\left(U_{-1}, B\right)$ is of the $\nu$-th kind (resp. classical). On the other hand, in our paper [1] we introduced the class of compact generalized Jordan triple systems, as a natural generalization of a similar concept for Jordan triple systems. Compact real simple Jordan triple systems were classified by Loos [10]. The second aim of this paper is to classify compact classical real simple generalized Jordan triple systems of the $\nu$-th kind, where $\nu=1,2$ (Theorems 4.1 and 4.2). Our result covers the above-mentioned result of Loos for classical ones. There are twelve families of compact classical simple generalized Jordan triple systems of the second kind. It turns out that the classification we are concerned is equivalent to that of simple graded Lie algebras of the $\nu$-th kind endowed with grade-reversing Cartan involutions (Theorem 3.14). In the course of this reduction, we make use of a result on the equivalence of pairs of an infinite-dimensional simple graded Lie algebra of type $\alpha_{0}$ and a gradereversing involution (Theorem 3.12). In $\S 5$ we will give a method of constructing noncompact generalized Jordan triple systems, starting from compact ones.

Throughout this paper, all objects are assumed to be defined over the real number field $\boldsymbol{R}$, unless otherwise stated. The complexification of a vector space $V$ (resp. a Lie algebra $g$ ) is denoted by $V^{c}$ (resp. $g^{c}$ ). $H$ denotes the field of quaternions. $Z$ denotes the ring of integers.

\section{§1. Gradations of real semisimple Lie algebras}

1.1. Let $\mathrm{g}=\sum_{k \in Z} \mathrm{~g}_{k}$ be a graded Lie algebra (or shortly GLA) over $\boldsymbol{R}$ with finite or infinite dimension. We always assume that $\operatorname{dim} g_{k}<\infty$ for all $k$ and that $\mathfrak{g}_{-1} \neq(0)$. The family of the subspaces $\left(g_{k}\right)$ is called a gradation in the Lie algebra $g$. We say that an element $E \in g$ is a characteristic element of the GLA $g$, if each subspace $g_{k}$ is the eigenspace of the operator ad $E$ for the eigenvalue $k$. Note that $E \in \mathfrak{g}_{0}$. Let $\left(\mathfrak{g}_{k}\right)$ and $\left(\mathfrak{g}_{k}^{\prime}\right)$ be two gradations in $\mathfrak{g}$. We say that $\left(\mathfrak{g}_{k}\right)$ and $\left(\mathfrak{g}_{k}^{\prime}\right)$ are isomorphic, if 
the GLA's $\mathfrak{g}=\sum \mathfrak{g}_{k}$ and $\mathfrak{g}=\sum \mathfrak{g}_{k}^{\prime}$ are isomorphic, that is, there exists an element $a \in$ Autg such that $a\left(\mathfrak{g}_{k}\right)=\mathfrak{g}_{k}^{\prime}$ for all $k$. A GLA $\mathfrak{g}=\sum_{k \in Z} \mathfrak{g}_{k}$ is said to be of the $\nu$-th kind $(\nu>0)$, if $g_{ \pm \nu} \neq(0)$ and $g_{k}=(0)$ for $|k|>\nu$. A finite-dimensional real semisimple GLA g has a grade-reversing Cartan involution [17], and consequently $g$ is of the $\nu$-th kind for some $\nu$. Note that the GLAg has a unique characteristic element $E$.

1.2. Let $\mathrm{g}=\sum \mathrm{g}_{k}$ be a real semisimple GLA of the $\nu$-th kind with characteristic element $E$, and let $\tau$ be a grade-reversing Cartan involution of $\mathfrak{g}$. Then we have $\tau(E)=-E$. Let $\mathfrak{g}=\mathfrak{f}+\mathfrak{p}$ be the Cartan decomposition by $\tau$, where $\left.\tau\right|_{\mathfrak{t}}=1$ and $\left.\tau\right|_{\mathfrak{p}}=-1$. Let us choose a maximal abelian subspace $\mathfrak{a}$ of $\mathfrak{p}$ containing $E$. We then have

$$
\mathfrak{a} \subset \mathfrak{g}_{0} \cap \mathfrak{p} \text {. }
$$

Let $\Delta$ be the (restricted) root system of $\mathfrak{g}$ with respect to $\mathfrak{a}$. We identify $\Delta$ with a subset of $a$ with respect to the inner product $\langle$,$\rangle on \mathfrak{a}$ induced by the Killing form of $\mathfrak{g}$, and we denote by $\mathfrak{g}^{\alpha}$ the root space for $\alpha \in \Delta$ in g. As a direct consequence of the decomposition of $\mathfrak{g}$ into root spaces, we have the following

LEMMA 1.1. Each graded subspace $\mathfrak{g}_{k}$ of $\mathfrak{g}$ is expressed as

$$
\begin{aligned}
& \mathfrak{g}_{0}=\mathfrak{c}(\mathfrak{a})+\sum_{\langle\alpha, E\rangle=0} \mathfrak{g}^{\alpha}, \\
& \mathfrak{g}_{k}=\sum_{\langle\alpha, E\rangle=k} \mathfrak{g}^{\alpha} \quad(k \neq 0,|k| \leqslant \nu),
\end{aligned}
$$

where $\mathfrak{c}(\mathfrak{a})$ is the centralizer of $\mathfrak{a}$ in $\mathfrak{g}$.

The above lemma shows that the gradation $\left(\mathfrak{g}_{k}\right)$ of $\mathfrak{g}$ gives rise to a partition of the root system $\Delta$ :

Also one has

$$
\begin{aligned}
& \Delta=\bigcup_{k=-\nu}^{\nu} \Delta_{k}, \\
& \Delta_{k}=\{\alpha \in \Delta:\langle\alpha, E\rangle=k\} .
\end{aligned}
$$

$$
\left(\Delta_{k}+\Delta_{l}\right) \cap \Delta \subset \Delta_{k+l}
$$

in particular $\Delta_{0}$ is a closed subsystem of $\Delta$. Let us choose a linear order in $\Delta$ in such a way that if $\alpha \in \Delta$ is positive then $\langle\alpha, E\rangle$ is non-negative. Let $\Pi$ be a simple root system of $\Delta$ relative to this linear order. Then we have

$$
I I \subset \bigcup_{k \geqslant 0} \Delta_{k}
$$


Putting $\Pi_{k}=\Pi \cap \Delta_{k}$, we get a partition of $\Pi$ :

$$
\Pi=\bigcup_{k=0}^{n} \Pi_{k},
$$

where $n$ is the number such that $\Pi_{n} \neq \varnothing, \Pi_{k}=\varnothing$ for every $k>n$. Note that $\Pi_{1}$ is not empty, since $\operatorname{dim} g_{1}=\operatorname{dim} g_{-1} \neq 0$. It is easy to see that $\Pi_{0}$ is a fundamental system for the root system $\Delta_{0}$.

Definition 1.2. Let $\Pi$ be a fundamental system of the root system $\Delta$. If $\Pi$ satisfies (1.5), then $\Pi$ is said to be compatible with the gradation $\left(\mathfrak{g}_{k}\right)$.

Let $W$ be the Weyl group of the root system $\Delta$ and $W_{0}$ be the Weyl group of the subsystem $\Delta_{0}$. Since the subalgebra $g_{0}$ of $g$ is stable under the Cartan involution $\tau$, it is reductive; $\tau$ induces a Cartan involution of the derived (semisimple) subalgebra $\left[g_{0}, g_{0}\right]$. We have the decomposition

$$
\mathfrak{g}_{0}=\mathfrak{f} \cap \mathfrak{g}_{0}+\mathfrak{p} \cap \mathfrak{g}_{0} .
$$

Since $\mathfrak{a}$ is a maximal abelian subspace of $\mathfrak{p} \cap g_{0}, \Delta_{0}$ is viewed as the (restricted) root system of $\mathfrak{g}_{0}$ with respect to $\mathfrak{a}$. Let $G=\operatorname{Ad} g$. Let $G_{0}$ and $K^{*}$ be the analytic subgroups of $G$ generated by $g_{0}$ and $\cap \cap g_{0}$, respectively. Then we have

$$
W_{0}=N_{K^{*}}(\mathfrak{a}) / C_{K^{*}}(\mathfrak{a}),
$$

where $N_{K^{*}}(\mathfrak{a})\left(\right.$ resp. $\left.C_{K^{*}}(\mathfrak{a})\right)$ is the normalizer (resp. centralizer) of $\mathfrak{a}$ in $K^{*}$.

Lemma 1.3. Let $I$ and $\Pi^{\prime}$ be two fundamental systems of $\Delta$ which are compatible with the gradation $\left(\mathrm{g}_{k}\right)$ of $\mathrm{g} . \quad$ Let $\Pi=\bigcup_{k=0}^{n} \Pi_{k}$ and $\Pi^{\prime}=\cup_{j=0}^{m} \Pi_{j}^{\prime}$ be the partitions of $\Pi$ and $\Pi^{\prime}$ given in (1.6). Then there exists $s \in W_{0}$ such that $s(\Pi)=\Pi^{\prime}$; in this case we have $n=m$ and $s\left(\Pi_{i}\right)=\Pi_{i}^{\prime}(1 \leqslant i \leqslant n)$.

Proof. For a fundamental system $\Omega$ of $\Delta$, we denote by $\Delta^{+}(\Omega)$ (resp. $\Delta^{-}(\Omega)$ ) the set of positive (resp. negative) roots in $\Delta$ with respect to the linear order determined by $\Omega$. We claim first that $\bigcup_{k \geqslant 1} \Delta_{k} \subset \Delta^{+}(I T)$. Let $\Pi=\left\{\alpha_{1}, \cdots, \alpha_{l}\right\}$. Choose a root $\alpha=\sum_{i} m_{i} \alpha_{i} \in \Delta_{k}(k \geqslant 1)$, where each $m_{i}$ is assumed to be non-zero. Since $\alpha \notin \Delta_{0}$, at least one $\alpha_{i}$ in the above expression are in $\Pi-\Pi_{0}$. Suppose $\alpha \in \Delta^{-}(I)$. Then each $m_{i}$ is negative, and hence we have $k=\langle\alpha, E\rangle=\sum_{j} m_{j}\left\langle\alpha_{j}, E\right\rangle\langle 0$. But this contradicts the assumption $k \geqslant 1$. Therefore we get $\alpha \in \Delta^{+}(I)$, which proves our claim. Similarly $\cup_{k \geqslant 1} \Delta_{k} \subset \Delta^{+}\left(\Pi^{\prime}\right)$ holds. Let us choose an element $s \in W_{0}$ which 
sends $\Pi_{0}$ to $\Pi_{0}^{\prime}$. By (1.8), $s$ has a representative in $G_{0}$ and so it comes from a grade-preserving inner automorphism of g. Consequently $s(E)=$ $E$. Therefore, for $\alpha_{k} \in \Pi_{i}(0 \leqslant i \leqslant n)$

$$
\left\langle s\left(\alpha_{k}\right), E\right\rangle=\left\langle s\left(\alpha_{k}\right), s(E)\right\rangle=\left\langle\alpha_{k}, E\right\rangle=i .
$$

Thus we have seen $s\left(\Pi_{i}\right) \subset \Delta_{i}(0 \leqslant i \leqslant n)$, which implies that the fundamental system $s(\Pi)$ of $\Delta$ is compatible with the gradation $\left(\mathfrak{g}_{k}\right)$. The same argument as for $\Pi$ shows that $\cup_{k \geqslant 1} \Delta_{k} \subset \Delta^{+}(s(I))$. Furthermore we see $s(\Pi) \cap \Delta_{0}=\Pi^{\prime} \cap \Delta_{0}$. Therefore it follows that $\Delta^{+}(s(\Pi))=\Delta^{+}\left(\Pi^{\prime}\right)$, which implies $s(\Pi)=\Pi^{\prime}$. Since $s$ is induced by a grade-preserving automorphism of $\mathfrak{g}$, we get $s\left(\Delta_{i}\right)=\Delta_{i}(1 \leqslant i \leqslant n)$, and consequently $s\left(\Pi_{i}\right)=\Pi_{i}^{\prime}(1 \leqslant i \leqslant n)$.

Lemma 1.4. Let $\tau_{1}$ and $\tau_{2}$ be two grade-reversing Cartan involutions of the GLA $\mathfrak{g}=\sum_{k} \mathfrak{g}_{k}$. Let $\mathfrak{g}=\mathfrak{f}_{i}+\mathfrak{p}_{i}$ be the Cartan decomposition by $\tau_{i}$ $(i=1,2)$, where $\left.\tau_{i}\right|_{t_{i}}=1$ and $\left.\tau_{i}\right|_{p_{i}}=-1$. Then there exists an element $X_{0}$ $\in \mathfrak{g}_{0} \cap \mathfrak{p}_{1} \cap \mathfrak{p}_{2}$ such that

$$
\left(\exp X_{0}\right) \tau_{2}\left(\exp \left(-X_{0}\right)\right)=\tau_{1},
$$

where the exponentials are taken in $G$.

Proof. Let $B$ be the Killing form of g. Put $B_{i}(X, Y)=-B\left(X, \tau_{i}(Y)\right)$, $i=1,2$. Then $B_{1}$ and $B_{2}$ are Autg-invariant inner products on $g$. Let $\operatorname{Pos}\left(\mathfrak{g}, B_{2}\right)$ (resp. Sym $\left(\mathfrak{g}, B_{2}\right)$ ) be the totality of positive definite symmetric (resp. symmetric) operators on $g$ relative to $B_{2}$. Since $\tau_{1}$ and $\tau_{2}$ are involutive, it follows that $\tau_{1} \tau_{2} \in \operatorname{Pos}\left(\mathfrak{g}, B_{2}\right)$. Let $C(E)$ be the algebraic subgroup of Aut $g$ consisting of all elements $g \in$ Aut $g$ which commute with ad $E$. Since $\tau_{i}$ is grade-reversing, we have $\tau_{i}(\operatorname{ad} E)=-(\operatorname{ad} E) \tau_{i}$. Therefore we have $\tau_{1} \tau_{2} \in C(E) \cap \operatorname{Pos}\left(\mathfrak{g}, B_{2}\right)$. By a result of Neher [11], there exists $X_{0} \in \operatorname{Lie} C(E) \cap \operatorname{Sym}\left(\mathfrak{g}, B_{2}\right)=\mathfrak{g}_{0} \cap \operatorname{Sym}\left(\mathfrak{g}, B_{2}\right)$ such that

$$
\begin{aligned}
& \tau_{i}\left(\operatorname{ad} X_{0}\right) \tau_{i}=-\operatorname{ad} X_{0}, \quad i=1,2, \\
& \left(\exp X_{0}\right) \tau_{2}\left(\exp \left(-X_{0}\right)\right)=\tau_{1} .
\end{aligned}
$$

In view of (1.11), we get $X_{0} \in \mathfrak{g}_{0} \cap \mathfrak{p}_{1} \cap \mathfrak{p}_{2}$.

1.3. Let $g$ be a real semisimple Lie algebra and $\Pi$ be a fundamental system of a restricted root system of $\mathrm{g}$. By a partition of $\Pi$ we mean a disjoint union $\Pi=\cup_{k=0}^{n} \Pi_{k}$ such that $\Pi_{1}$ and $\Pi_{n}$ are not empty. Some of the subsets $\Pi_{i}$ may be empty. The partition is sometimes denoted by $\left(\Pi_{0}, \Pi_{1}, \cdots, \Pi_{n}\right)$. 
Definition 1.5. Let $\mathfrak{g}$ and $\mathfrak{g}^{\prime}$ be real semisimple Lie algebras, and let $\Pi$ and $\Pi^{\prime}$ be fundamental systems of restricted root systems of $\mathfrak{g}$ and $\mathrm{g}^{\prime}$, respectively. Partitions $\left(\Pi_{0}, \cdots, \Pi_{n}\right)$ of $\Pi$ and $\left(\Pi_{0}^{\prime}, \cdots, \Pi_{m}^{\prime}\right)$ of $\Pi^{\prime}$ are said to be equivalent, if $n=m$ and if there exists an isomorphism $\varphi$ of the Dynkin diagram of $\Pi$ to that of $\Pi^{\prime}$ sending $\Pi_{i}$ to $\Pi_{i}^{\prime}(0 \leqslant i \leqslant n)$.

The following theorem is a criterion as to whether two real semisimple GLA's are isomorphic.

TheOREM 1.6. Let $\mathrm{g}=\sum_{k} \mathfrak{g}_{k}$ and $\mathrm{g}^{\prime}=\sum_{k} \mathfrak{g}_{k}^{\prime}$ be real semisimple GLA's of the $\nu$-th kind, and let $\Pi$ and $\Pi^{\prime}$ be, respectively, fundamental systems of restricted root systems of $\mathfrak{g}$ and $\mathfrak{g}^{\prime}$ compatible with the gradations. Let $\left(\Pi_{0}, \cdots, \Pi_{n}\right)$ and $\left(\Pi_{0}^{\prime}, \cdots, \Pi_{m}^{\prime}\right)$ be the partitions of $\Pi$ and $\Pi^{\prime}$ given in (1.6). If the two GLA's are isomorphic, then the above two partitions are equivalent. The converse is true, if the Lie algebras $\mathrm{g}$ and $\mathrm{g}^{\prime}$ are isomorphic.

Proof. Let $E$ (resp. $E^{\prime}$ ) be the characteristic element of the GLA $g$ (resp. $\left.g^{\prime}\right)$. Let $\Delta$ (resp. $\Delta^{\prime}$ ) be the restricted root system of $g$ (resp. $\mathfrak{g}^{\prime}$ ) with $\Pi$ (resp. $\left.\Pi^{\prime}\right)$ as a fundamental system. Since $\Pi$ and $\Pi^{\prime}$ are compatible with the gradations, we can suppose that $\Delta$ (resp. $\Delta^{\prime}$ ) is the root system with respect to a maximal abelian subspace $a$ (resp. $\left.\mathfrak{a}^{\prime}\right)$ of the $(-1)$-eigenspace $\mathfrak{p}$ (resp. $\left.\mathfrak{p}^{\prime}\right)$ in $\mathfrak{g}\left(\right.$ resp. $\left.\mathfrak{g}^{\prime}\right)$ under a grade-reversing Cartan involution $\tau$ (resp. $\tau^{\prime}$ ) of $\mathfrak{g}$ (resp. $\mathfrak{g}^{\prime}$ ) satisfying $E \in \mathfrak{a}$ (resp. $E^{\prime} \in \mathfrak{a}^{\prime}$ ). Let $\mathfrak{f}$ and $\mathfrak{f}^{\prime}$ be the $(+1)$-eigenspaces in $\mathfrak{g}$ and $\mathfrak{g}^{\prime}$ under $\tau$ and $\tau^{\prime}$, respectively. Now let $\varphi$ be a grade-preserving isomorphism of $\mathfrak{g}$ onto $\mathfrak{g}^{\prime} . \varphi \tau \varphi^{-1}$ is a grade-reversing Cartan involution of $\mathfrak{g}^{\prime}$. By Lemma 1.4 , one can find an element $X_{0} \in \mathfrak{g}_{0}^{\prime}$ such that $\varphi_{1}:=\left(\exp X_{0}\right) \varphi$ satisfies

$$
\varphi_{1} \tau=\tau^{\prime} \varphi_{1}
$$

where the exponential is taken in the adjoint group of $\mathrm{g}^{\prime} \cdot \varphi_{1}$ is a gradepreserving isomorphism, since $X_{0} \in \mathfrak{g}_{0}^{\prime}$. By (1.13) we have

$$
\varphi_{1}(\mathfrak{l})=\mathfrak{l}^{\prime}, \quad \varphi_{1}(\mathfrak{p})=\mathfrak{p}^{\prime} .
$$

Next we claim that there exists an element $X_{1} \in \mathfrak{f}^{\prime} \cap \mathfrak{g}_{0}^{\prime}$ such that $\varphi_{:}:=$ $\left(\exp X_{1}\right) \varphi_{1}$ satisfies

$$
\varphi_{2}(\mathfrak{a})=\mathfrak{a}^{\prime} .
$$

The reductive subalgebra $\mathfrak{g}_{0}^{\prime}$ of $\mathfrak{g}^{\prime}$ can be decomposed by $\tau^{\prime}$ :

$$
\mathfrak{g}_{0}^{\prime}=\mathfrak{f}^{\prime} \cap \mathfrak{g}_{0}^{\prime}+\mathfrak{p}^{\prime} \cap \mathfrak{g}_{0}^{\prime} .
$$


Since $\varphi_{1}$ is grade-preserving, both $\varphi_{1}(\mathfrak{a})$ and $\mathfrak{a}^{\prime}$ are maximal abelian subspaces of $\mathfrak{p}^{\prime} \cap \mathfrak{g}_{0}^{\prime}$ (cf. (1.1)). Therefore, for the decomposition (1.16), one can find $X_{1} \in \mathfrak{f}^{\prime} \cap \mathfrak{g}_{0}^{\prime}$ such that $\varphi_{2}(\mathfrak{a})=\left(\exp X_{1}\right) \varphi_{1}(\mathfrak{a})=\mathfrak{a}^{\prime}$. Note that $\varphi_{2}$ is still grade-preserving. Let $\Delta=\cup_{k=-\nu}^{\nu} \Delta_{k}$ and $\Delta^{\prime}=\bigcup_{k=-\nu}^{\nu} \Delta_{k}^{\prime}$ be the partitions given by (1.3). By (1.15) we have $\varphi_{2}(\Delta)=\Delta^{\prime}$ and moreover

$$
\varphi_{2}\left(\Delta_{k}\right)=\Delta_{k}^{\prime}, \quad|k| \leqslant \nu .
$$

From this it follows that the fundamental system $\varphi_{2}(\Pi)$ of $\Delta^{\prime}$ is compatible with the gradation $\left(\mathfrak{g}_{k}^{\prime}\right)$. By (1.8) and Lemma 1.3, there exists an element $X_{2} \in \mathfrak{I}^{\prime} \cap \mathfrak{g}_{0}^{\prime}$ such that $\varphi_{3}:=\left(\exp X_{2}\right) \varphi_{2}$ sends $\Pi$ to $\Pi^{\prime}$ and $\Pi_{i}$ to $\Pi_{i}^{\prime}(0 \leqslant i \leqslant$ $n=m)$.

To prove the converse, let $\psi$ be an isomorphism of $\left(\Pi_{0}, \cdots, \Pi_{n}\right)$ to $\left(\Pi_{0}^{\prime}, \cdots, \Pi_{n}^{\prime}\right)$. Under the assumption, $\psi$ extends to an isomorphism of $g$ onto $\mathfrak{g}^{\prime}$, denoted again by $\psi$. Let $\Pi=\left\{\alpha_{1}, \cdots, \alpha_{l}\right\}$ and $\Pi^{\prime}=\left\{\beta_{1}, \cdots, \beta_{l}\right\}$. The characteristic elements $E$ and $E^{\prime}$ are uniquely determined by the equations

$$
\left\langle E, \alpha_{i}\right\rangle=k, \quad\left\langle E^{\prime}, \beta_{j}\right\rangle=k
$$

for $\alpha_{i} \in \Pi_{k}, 0 \leqslant k \leqslant n, 1 \leqslant i \leqslant l$, and for $\beta_{j} \in \Pi_{k}^{\prime}, 0 \leqslant k \leqslant n, 1 \leqslant j \leqslant l$, where $\langle$,$\rangle denotes the inner products defined by the Killing forms of g$ and $g^{\prime}$. We may assume $\psi\left(\alpha_{i}\right)=\beta_{i}(1 \leqslant i \leqslant l)$ by renumbering roots in $\Pi^{\prime}$. Then we have

$$
\left\langle\psi(E), \beta_{i}\right\rangle=\left\langle\psi(E), \psi\left(\alpha_{i}\right)\right\rangle=\left\langle E, \alpha_{i}\right\rangle=k
$$

for $\alpha_{i} \in \Pi_{k}$, or equivalently $\beta_{i} \in \Pi_{k}^{\prime}$. Comparing (1.19) with (1.18), we conclude $\psi(E)=E^{\prime}$.

The following is a classification theorem for gradations in a semisimple Lie algebra.

Theorem 1.7. Let $\mathrm{g}$ be a real semisimple Lie algebra and $\Pi$ be a fixed fundamental system of a fixed restricted root system $\Delta$ of $\mathrm{g}$. Let $\mathscr{G}$ be the set of isomorphism classes of gradations in $\mathrm{g}$ and let $\mathscr{P}$ be the set of equivalence classes of partitions of $\Pi$ under the automorphism group of the Dynkin diagram of $\Pi$. Then there exists a bijection $\Phi$ of $\mathscr{G}$ to $\mathscr{P}$.

Proof. Choose a gradation $\left(\mathfrak{g}_{k}\right)$ of $\mathfrak{g}$. To this gradation one can associate a compatible fundamental system $\Pi^{(1)}$ (cf. Definition 1.2) and the compatible partition $\left(\Pi_{0}^{(1)}, \cdots, \Pi_{n}^{(1)}\right)$ of $\Pi^{(1)}$ (cf. (1.6)). According to Satake [13], there exists $a \in \operatorname{Adg}$ such that $a\left(\Pi^{(1)}\right)=\Pi$. Put $\Pi_{k}=a\left(\Pi_{k}^{(1)}\right)$. Then 
$a$ gives an equivalence between the two partitions $\left(\Pi_{0}^{(1)}, \cdots, \Pi_{n}^{(1)}\right)$ and $\left(\Pi_{0}, \cdots, \Pi_{n}\right)$. Choose another gradation $\left(g_{k}^{\prime}\right)$ of $g$ which is isomorphic to $\left(\mathfrak{g}_{k}\right)$ under $\varphi \in$ Aut $g$. To $\left(\mathfrak{g}_{k}^{\prime}\right)$ there correspond a compatible fundamental system $\Pi^{(2)}$ and the compatible partition $\left(\Pi_{0}^{(2)}, \cdots, \Pi_{m}^{(2)}\right)$. There exists $b \in$ Ad $g$ such that $b\left(\Pi^{(2)}\right)=\Pi$. Let $\Pi_{k}^{\prime}=b\left(\Pi_{k}^{(2)}\right)$. Then $b$ gives an equivalence of $\left(\Pi_{0}^{(2)}, \cdots, \Pi_{m}^{(2)}\right)$ to $\left(\Pi_{0}^{\prime}, \cdots, \Pi_{m}^{\prime}\right)$. From Theorem 1.6 and its proof it follows that $m=n$ and that $\varphi$ can be modified to give an isomorphism $\tilde{\varphi}$ (still contained in Autg) of $\Pi^{(2)}$ to $\Pi^{(1)}$ which sends $\left(\Pi_{0}^{(2)}, \cdots, \Pi_{n}^{(2)}\right)$ to $\left(\Pi_{0}^{(1)}, \cdots, \Pi_{n}^{(1)}\right)$. Therefore $b \tilde{\varphi}^{-1} a^{-1}$ induces an automorphism of the Dynkin diagram of $\Pi$ which sends $\left(\Pi_{0}, \cdots, \Pi_{n}\right)$ to $\left(\Pi_{0}^{\prime}, \cdots, \Pi_{n}^{\prime}\right)$. Thus we can define the mapping $\Phi$ by putting

$$
\Phi\left(\left[\left(\mathfrak{g}_{k}\right)\right]\right)=\left[\left(\Pi_{0}, \cdots, \Pi_{n}\right)\right],
$$

where [ ] denotes the isomorphism (or equivalence) class. That $\Phi$ is injective follows from Theorem 1.6. We want to prove the surjectivity of $\Phi$. Let $\Pi=\left\{\alpha_{1}, \cdots, \alpha_{l}\right\}$ and let $\left(\Pi_{0}, \cdots, \Pi_{n}\right)$ be a partition of $\Pi$. We write $\alpha \in \Delta$ in the form $\alpha=\sum_{i=1}^{l} m_{i}(\alpha) \alpha_{i}$. For the partition $\left(\Pi_{0}, \cdots, \Pi_{n}\right)$, let us define an integer-valued function $h_{I I}$ on $\Delta$ in the following way:

$$
h_{I I}(\alpha)=\sum_{\alpha i \in \Pi_{1}} m_{i}(\alpha)+2 \sum_{\alpha_{j} \in \Pi_{2}} m_{j}(\alpha)+\cdots+n \sum_{\alpha_{k} \in \Pi_{n}} m_{k}(\alpha) .
$$

Let $\alpha, \beta \in \Delta$. If $\alpha+\beta \in \Delta$, then

$$
h_{I I}(\alpha+\beta)=h_{I I}(\alpha)+h_{I I}(\beta) .
$$

Let us put

$$
\begin{aligned}
& \mathfrak{g}_{p}=\sum_{n_{\Pi^{(\alpha)}=p}} \mathfrak{g}^{\alpha}, \quad p \neq 0, p \in Z, \\
& \mathfrak{g}_{0}=\mathfrak{c}(\mathfrak{a})+\sum_{n_{I^{(\alpha)}}=0} \mathfrak{g}^{\alpha},
\end{aligned}
$$

where $\mathfrak{a}$ is the abelian subspace of $\mathfrak{g}$ on which $\Delta$ is defined. Then we have $\mathfrak{g}=\sum \mathfrak{g}_{p}$. By (1.22), $\left(\mathfrak{g}_{p}\right)$ is a gradation of $\mathfrak{g}$. Put

$$
\Delta_{p}=\left\{\alpha \in \Delta: h_{I I}(\alpha)=p\right\}, \quad p \in Z .
$$

Then we get $\Pi_{p}=\Pi \cap \Delta_{p}$. This implies that $\Phi\left(\left[\left(\mathfrak{g}_{p}\right)\right]\right)=\left[\left(\Pi_{0}, \cdots, \Pi_{n}\right)\right]$.

Remark 1.8. The partition (1.6) has been considered by Kac [5] for the complex semisimple case and is called the characteristic of the gradation $\left(g_{k}\right)$. We will use this terminology for the real semisimple case.

Definition 1.9. The gradation given in (1.23) is called the gradation 
defined by the partition $\left(\Pi_{0}, \cdots, \Pi_{n}\right)$.

Remark 1.10. Let $\mathfrak{g}=\sum \mathfrak{g}_{k}$ be a real semisimple GLA of the $\nu$-th kind with characteristic element $E$, and let $\Pi$ be a fundamental system compatible with the gradation $\left(\mathfrak{g}_{k}\right)$. Let $\Pi=\cup_{k=0}^{n} \Pi_{k}$ be the partition given in (1.6). Then it follows easily that $h_{\Pi}(\alpha)=\langle\alpha, E\rangle$ holds, and hence the gradation $\left(\mathfrak{g}_{k}\right)$ coincides with the gradation defined by the partition $\left(\Pi_{0}, \cdots, \Pi_{n}\right)$.

\section{§2. Gradations of type $\alpha_{0}$}

2.1. Definition 2.1. Let $\mathfrak{g}=\sum_{k \in \boldsymbol{Z}} \mathfrak{g}_{k}$ be a real GLA with $\operatorname{dim} \mathfrak{g} \leqslant \infty$. We say that $\mathfrak{g}$ is of type $\alpha_{0}$ if the following conditions are satisfied:

$$
\mathfrak{g}_{-k-1}=\left[\mathfrak{g}_{-k}, \mathfrak{g}_{-1}\right], \quad \mathfrak{g}_{k+1}=\left[\mathfrak{g}_{k}, \mathfrak{g}_{1}\right] \quad(k \geqslant 1) .
$$

Lemma 2.2. Let $\mathfrak{g}=\sum \mathfrak{g}_{k}$ be a real semisimple GLA of the $\nu$-th kind, and let $\Pi=\cup_{k=0}^{n} \Pi_{k}$ be the characteristic of the gradation $\left(\mathfrak{g}_{k}\right)$. If $\left(\mathfrak{g}_{k}\right)$ is of type $\alpha_{0}$, then $\Pi_{k}=\varnothing$ for $k \geqslant 2$.

Proof. Let $E$ be the characteristic element of the gradation $\left(\mathfrak{g}_{k}\right)$. Note that $\Pi_{1} \neq \varnothing$ (cf. 1.2). Choose a root $\alpha_{i} \in \Pi-\Pi_{0}$. Suppose that $\left\langle\alpha_{i}, E\right\rangle=$ $k>1$. Since $\left(\mathfrak{g}_{k}\right)$ is of type $\alpha_{0}$, we have

$$
\mathrm{g}^{\alpha_{i}} \subset \mathrm{g}_{k}=\left[\mathfrak{g}_{k-1}, \mathfrak{g}_{1}\right]=\sum^{\prime}\left[\mathfrak{g}^{\beta}, \mathfrak{g}^{r}\right],
$$

where the sum $\Sigma^{\prime}$ is taken over the roots $\beta$ and $\gamma$ such that $\langle\beta, E\rangle=k$ $-1,\langle\gamma, E\rangle=1$. If $\left[\mathrm{g}^{\beta}, \mathrm{g}^{\gamma}\right] \neq(0)$, then $\beta+\gamma$ is a root. Taking account of (1.2), we conclude that there exist two positive roots $\beta, \gamma$ such that $\alpha_{i}=$ $\beta+\gamma,\langle\beta, E\rangle=k-1$ and $\langle\gamma, E\rangle=1$. This contradicts the fact that $\alpha_{i}$ is simple. Therefore we have $k=1$, or equivalently $\alpha_{i} \in \Pi_{1}$.

Lemma 2.3. Let $\mathrm{g}$ be a complex semisimple Lie algebra, and $\Pi$ be a fundamental system of a root system $\Delta$ of g. Let $\left(\Pi_{0}, \Pi_{1}\right)$ be a partition of II. Then the gradation $\left(\mathfrak{g}_{k}\right)$ defined by $\left(\Pi_{0}, \Pi_{1}\right)$ (cf. Definition 1.9) is of type $\alpha_{0}$.

Proof. Let $\Pi=\left\{\alpha_{1}, \cdots, \alpha_{l}\right\}$ and $\Pi_{1}=\left\{\alpha_{i_{1}}, \cdots, \alpha_{i_{s}}\right\}$. We write $\alpha \in \Delta$ in the form $\sum_{i=1}^{l} m_{i}(\alpha) \alpha_{i}$. Then the function $h_{\Pi}$ in (1.21) for the partition $\left(\Pi_{0}, \Pi_{1}\right)$ is given by

$$
h_{\Pi}(\alpha)=\sum_{k=1}^{s} m_{i_{k}}(\alpha)
$$


We define the function $h$ on $\Delta$ by

$$
h(\alpha)=\sum_{i=1}^{l} m_{i}(\alpha) .
$$

Let $\Delta=\cup_{k} \Delta_{k}$ be the partition (1.3) induced by the gradation $\left(\mathrm{g}_{k}\right)$. If we put $\Delta_{p}^{(k)}=\left\{\alpha \in \Delta_{p}: h(\alpha)=k\right\}$, then we have a partition of $\Delta_{p}$ :

$$
\Delta_{p}=\bigcup_{k \geqslant p} \Delta_{p}^{(k)} \quad(p>0) .
$$

We claim

$$
\mathfrak{g}^{\beta} \subset\left[\mathfrak{g}_{1}, \mathfrak{g}_{p-1}\right], \quad \beta \in \Delta_{p} \quad(p \geqslant 2) .
$$

We want to prove this by induction on $k$ in (2.5). Let us take $\beta \in \Delta_{p}^{c p}$ first. We have then $h(\beta)=h_{I I}(\beta)=p$, which implies that $m_{i}(\beta)=0$ for $i \neq i_{1}, \cdots, i_{s}$. Hence one can write $\beta$ as

$$
\beta=\sum_{k=1}^{s} m_{i_{k}}(\beta) \alpha_{i_{k}} .
$$

Since $\beta$ is in $\Delta_{p}(p \geqslant 2), \beta$ is positive but not simple. Consequently, there exists a root $\alpha_{i} \in \Pi$ such that $\beta-\alpha_{i}$ is a root. Therefore, in view of the expression (2.7), we have $\alpha_{i} \notin \Pi_{0}$, that is, $\alpha_{i}$ coincides with one of $\alpha_{i_{t}}$ $(1 \leqslant t \leqslant s)$, say $\alpha_{i_{k}}$. Set $\gamma=\beta-\alpha_{i_{k}} \in \Delta$. We have then $h_{I I}(\gamma)=h_{I I}(\beta)-1$ $=p-1$. This implies $\gamma \in \Delta_{p-1}$. Therefore we obtain $\mathfrak{g}^{\beta}=\left[\mathfrak{g}^{\alpha i_{k}}, \mathfrak{g}^{r}\right] \subset\left[\mathfrak{g}_{1}, \mathfrak{g}_{p-1}\right]$, which proves (2.6) for $\beta \in \Delta_{p}^{(p)}$.

Suppose next that (2.6) is valid for all $\alpha \in \Delta_{p}^{(m)}$, and choose $\beta \in \Delta_{p}^{(m+1)}$. By the same reason as above, $\beta$ is positive but not simple. We have one of the following two situations: a) There exists $\alpha_{i_{k}} \in \Pi_{1}$ such that $\beta-\alpha_{i_{k}}$ $\in \Delta$, b) there exists $\alpha_{j} \in \Pi_{0}$ such that $\beta-\alpha_{j} \in \Delta$. In the case a) we proceed as above to get the assertion (2.6). Suppose that b) occurs. Let $\gamma=\beta-\alpha_{j} \in \Delta$. Then we have $h_{\Pi}(\gamma)=h_{\Pi}(\beta)=p$ and $h(\gamma)=h(\beta)-1=m$. Hence $\gamma \in \Delta_{p}^{(m)}$. By the assumption of the induction, we get $\mathrm{g}^{\gamma} \subset\left[\mathfrak{g}_{1}, \mathrm{~g}_{p-1}\right]$. Therefore we obtain

$$
\mathfrak{g}^{\beta}=\left[\mathfrak{g}^{\alpha}, \mathfrak{g}^{\tau}\right] \subset\left[\mathfrak{g}^{\alpha},\left[\mathfrak{g}_{1}, \mathfrak{g}_{p-1}\right]\right] \subset\left[\mathfrak{g}_{0},\left[\mathfrak{g}_{1}, \mathfrak{g}_{p-1}\right]\right] \subset\left[\mathfrak{g}_{1}, \mathfrak{g}_{p-1}\right]
$$

Thus we have proved (2.6). The second equality of (2.1) is a direct consequence of (2.6). The first one in (2.1) is immediately obtained from the second by applying a grade-reversing Cartan involution.

2.2. Let $\mathfrak{g}=\sum \mathrm{g}_{k}$ be a real semisimple GLA of the $\nu$-th kind. We come back to the situation in 1.2 and preserve notations there. Let $\mathfrak{h}$ be 
a Cartan subalgebra of $\mathfrak{g}$ containing $\mathfrak{a}$. Then one can write $\mathfrak{h}=\mathfrak{h}^{+}+\mathfrak{a}$, where $\mathfrak{h}^{+}=\mathfrak{h} \cap \mathfrak{f}$. Let $\tilde{\Delta}$ be the root system of the complexification $\mathfrak{g}^{c}$ of $\mathrm{g}$ with respect to the Cartan subalgebra $\mathfrak{h}^{c}(=$ the complexification of $\mathfrak{h})$. $\mathfrak{h}_{0}:=i \mathfrak{h}^{+}+\mathfrak{a}$ is the real part of $\mathfrak{h}^{c}$. We identify $\tilde{\Delta}$ with a subset of $\mathfrak{h}_{0}$ via the inner product $\langle$,$\rangle defined by the Killing form of \mathrm{g}^{c}$. Put $\tilde{\Delta}_{\bullet}$ $=\tilde{\Delta} \cap i \mathrm{~h}^{+}$. Let $\sigma$ be the conjugation of $\mathrm{g}^{c}$ with respect to $\mathrm{g}$, and let $\tilde{\Pi}$ be the $\sigma$-fundamental system of $\tilde{\Delta}$ [13]. Let $\widetilde{\sigma}$ be the orthogonal projection of $\mathfrak{h}_{0}$ onto $\mathfrak{a}$ with respect to $\langle,\rangle . \Delta:=\widetilde{\sigma}\left(\tilde{\Delta}-\tilde{\Delta}_{\bullet}\right)$ is a restricted root system (or the root system of $\mathfrak{g}$ with respect to $\mathfrak{a}$ ). Let us consider the complexified GLA of $g$ :

$$
\mathfrak{g}^{c}=\sum_{k} \mathfrak{g}_{k}^{c}
$$

where $\mathfrak{g}_{k}^{C}$ is the complexification of $\mathfrak{g}_{k}$.

LEMma 2.4. (i) The characteristic element $E$ of $\mathfrak{g}$ is also that of $\mathfrak{g}^{c}$. (ii) The following partition is valid:

$$
\tilde{\Delta}=\bigcup_{k} \tilde{\Delta}_{k},
$$

where $\tilde{\Delta}_{k}=\{\alpha \in \tilde{\Delta}:\langle\alpha, E\rangle=k\}$. (iii) We have

$$
\begin{aligned}
& \tilde{\Delta}_{0}=\left(\varpi^{-1}\left(\Delta_{0}\right) \cap \tilde{\Delta}\right) \cup \tilde{\Delta}_{\bullet}, \\
& \tilde{\Delta}_{k}=\widetilde{\varpi}^{-1}\left(\Delta_{k}\right) \cap \tilde{\Delta} \quad(k \neq 0) .
\end{aligned}
$$

(iv) The subspace $\mathfrak{g}_{k}^{C}$ are expressed as

$$
\begin{aligned}
& \mathfrak{g}_{0}^{C}=\mathfrak{h}^{c}+\sum_{\alpha \in \tilde{I}_{0}} \tilde{\mathfrak{g}}^{\alpha}, \\
& \mathfrak{g}_{k}^{C}=\sum_{\alpha \in \tilde{\Omega}_{k}} \tilde{\mathfrak{g}}^{\alpha} \quad(k \neq 0),
\end{aligned}
$$

where $\tilde{\mathfrak{g}}^{\alpha}$ is the root space in $\mathfrak{g}^{c}$ corresponding to $\alpha \in \tilde{\Delta}$.

Proof. The assertions (i), (ii) are immediate. Let $\alpha \in \tilde{\Delta}_{0} . \quad \widetilde{\sigma}(\alpha)=0$ if

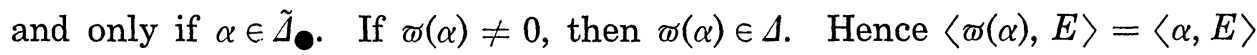
$=0$, which implies $\varpi(\alpha) \in \Delta_{0}$. Thus the inclusion $\subset$ in (2.11) was obtained. Similarly we have the converse inclusion. (2.12) can be proved analogously. By (1.2) we have

$$
\mathfrak{g}_{0}^{c}=\mathfrak{c}(\mathfrak{a})^{c}+\sum_{r \in \Delta_{0}}\left(\mathfrak{g}^{r}\right)^{c} .
$$

From the expression of $\mathfrak{c}(\mathfrak{a})$ in terms of the roots in $\tilde{\Delta}_{\bullet}$ (cf. [15]), we see 
that $\mathfrak{c}(\mathfrak{a})^{c}=\mathfrak{h}^{c}+\sum_{\alpha \in \tilde{I}} . \tilde{\mathfrak{g}}^{\alpha}$. On the other hand we have $\left(\mathfrak{g}^{\gamma}\right)^{c}=\sum^{\prime} \tilde{\mathfrak{g}}^{\alpha}$, where the sum $\Sigma^{\prime}$ is taken over the roots $\alpha \in \tilde{\Delta}$ satisfying $\widetilde{\sigma}(\alpha)=\gamma$. Therefore, from (2.11) and (2.15) we obtain (2.13). Similarly we have (2.14).

We can choose a $\sigma$-fundamental system $\tilde{\Pi}=\left\{\alpha_{1}, \cdots, \alpha_{s}\right\}$ of $\tilde{\Delta}$ in such a way that the relation

$$
\left\langle\alpha_{i}, E\right\rangle \geqslant 0 \quad(1 \leqslant i \leqslant s)
$$

is satisfied. $\tilde{\Pi}$ is compatible with the gradation $\left(\mathfrak{g}_{k}^{c}\right)$ of $\mathfrak{g}^{c}$. Let $\tilde{\Pi}_{\bullet}=\tilde{\Pi}$ $\cap \tilde{\Delta}_{\bullet}$. Then $\Pi=\widetilde{\varpi}\left(\tilde{\Pi}-\tilde{\Pi}_{\bullet}\right)$ is a fundamental system of $\Delta$. By (2.16) we have $\left\langle\gamma_{i}, E\right\rangle \geqslant 0$ for each $\gamma_{i} \in \Pi$, and so $\Pi$ is compatible with the gradation $\left(\mathfrak{g}_{k}\right)$ of $\mathfrak{g}$. Let $\tilde{\Pi}_{i}=\tilde{\Pi} \cap \tilde{\Delta}_{i}$ and $\Pi_{i}=\Pi \cap \Delta_{i}$. Then the following lemma is easily seen.

$$
\begin{array}{ll}
\text { Lemma 2.5. } & \tilde{\Pi}_{0}=\left(\widetilde{\varpi}^{-1}\left(\Pi_{0}\right) \cap \tilde{\Pi}\right) \cup \tilde{\Pi}_{\bullet}, \\
& \tilde{\Pi}_{k}=\widetilde{\varpi}^{-1}\left(\Pi_{k}\right) \cap \tilde{\Pi} \quad(k \neq 0) .
\end{array}
$$

In particular, the number of $\tilde{\Pi}_{i}$ 's in the partition $\tilde{\Pi}=\cup_{i} \tilde{\Pi}_{i}$ is equal to the number of $\Pi_{i}$ 's in the partition $\Pi=\cup_{i} \Pi_{i}$.

The next theorem gives a characterization of the gradations of type $\alpha_{0}$ in terms of their characteristics.

TheOREM 2.6. Let $\mathrm{g}=\sum \mathrm{g}_{k}$ be a real semisimple GLA of the $\nu$-th kind, and $\Pi=\cup_{k=0}^{n} \Pi_{k}$ be the characteristic of the gradation $\left(\mathfrak{g}_{k}\right)$. Then $\left(\mathfrak{g}_{k}\right)$ is of type $\alpha_{0}$ if and only if $\Pi_{k}=\varnothing$ for every $k \geqslant 2$.

Proof. Suppose that $\Pi_{k}=\varnothing(k \geqslant 2)$. Consider the GLA $\mathrm{g}^{c}=\sum \mathrm{g}_{k}^{c}$, and let $\tilde{\Pi}=\cup_{k=0}^{n} \tilde{\Pi}_{k}$ be the characteristic of the gradation $\left(\mathrm{g}_{k}^{C}\right)$. Then, by Lemma 2.5 and Theorem 1.6, we get $\tilde{\Pi}_{k}=\varnothing(k \geqslant 2)$. Lemma 2.3 and Remark 1.10 now imply that $\left(\mathfrak{g}_{k}^{C}\right)$ is of type $\alpha_{0}$. Hence, for $k \geqslant 1$, we have $\mathfrak{g}_{k+1}^{C}=\left[\mathfrak{g}_{1}^{c}, \mathfrak{g}_{k}^{C}\right]=\left[\mathfrak{g}_{1}, \mathfrak{g}_{k}\right]^{C}$, from which it follows that $\mathfrak{g}_{k+1}=\left[\mathfrak{g}_{1}, \mathfrak{g}_{k}\right]$. The converse assertion has been proved in Lemma 2.2 .

Let $\Pi$ be a fundamental system of a restricted root system of a real semisimple Lie algebra g. Two subsets $\Omega_{1}, \Omega_{2} \subset \Pi$ are said to be equivalent, if there exists an automorphism $a$ of the Dynkin diagram of $\Pi$ sending $\Omega_{1}$ to $\Omega_{2}$. The equivalence class of $\Omega_{1}$ is denoted by $\left[\Omega_{1}\right]$. Combining Theorem 2.6 with Theorem 1.7 we have the following

TheOREM 2.7. Let $\mathrm{g}$ be a real semisimple Lie algebra, and let $\Pi$ be a fundamental system of a restricted root system of $\mathrm{g}$. Let $\mathscr{G}_{\alpha_{0}}$ be the set 
of isomorphism classes of gradations of type $\alpha_{0}$ in $\mathfrak{g}$, and let $\mathscr{P}_{\alpha_{0}}$ be the set of equivalence classes of all non-empty subsets of $\Pi$. Then there exists a bijection $\Phi_{\alpha_{0}}$ of $\mathscr{G}_{\alpha_{0}}$ to $\mathscr{P}_{\alpha_{0}}$.

Proof. Let $\left[\left(\mathfrak{g}_{k}\right)\right]$ denote the isomorphism class of the gradation $\left(\mathfrak{g}_{k}\right)$ of $\mathfrak{g}$. By Theorem 2.6, the characteristic of $\left(\mathfrak{g}_{k}\right)$ is of the form $\Pi=\Pi_{0}$ $\cup \Pi_{1}$ (See also Theorem 1.6). By Theorem 1.6 we may define $\Phi_{\alpha_{0}}$ to be

$$
\Phi_{\alpha_{0}}\left(\left[\left(\mathfrak{g}_{k}\right)\right]\right)=\left[\Pi_{1}\right] .
$$

Then Theorem 1.7 shows that $\Phi_{\alpha_{0}}$ is bijective.

Now we will assume $g$ to be real simple. Let $\mathscr{G}$ be the same as in Theorem 1.7, and let $\mathscr{G}^{(\nu)}$ be the subset of $\mathscr{G}$ consisting of isomorphism classes of all gradations of the $\nu$-th kind in $g$. Let $\mathscr{G}_{\alpha_{0}}^{(\nu)}=\mathscr{G}^{(\nu)} \cap \mathscr{G}_{\alpha_{0}}$.

LEMMA 2.8. $\mathscr{G}^{(\nu)}=\mathscr{G}_{\alpha_{0}}^{(\nu)}$ holds for $\nu=1,2$.

Proof. The case $\nu=1$ is trivial. For $\nu=2$, see Tanaka [16].

Let $\Pi=\left\{\alpha_{1}, \cdots, \alpha_{l}\right\}$ be a fundamental system of a restricted root system $\Delta$ of $\mathrm{g}$, and $\vartheta$ be the dominant root. We write $\vartheta=\sum_{i=1}^{l} m_{i}(\vartheta) \alpha_{i}$. Let

$$
\begin{aligned}
\mathscr{P}^{(1)}= & \left\{\left[\left\{\alpha_{i}\right\}\right]: m_{i}(\vartheta)=1 \quad(1 \leqslant i \leqslant l)\right\}, \\
\mathscr{G}^{(2)}= & \left\{\left[\left\{\alpha_{i}\right\}\right]: m_{i}(\vartheta)=2 \quad(1 \leqslant i \leqslant l)\right\} \\
& \bigcup\left\{\left[\left\{\alpha_{i}, \alpha_{j}\right\}\right]: m_{i}(\vartheta)=m_{j}(\vartheta)=1 \quad(1 \leqslant i \neq j \leqslant l)\right\} .
\end{aligned}
$$

The next theorem gives the classification of real simple GLA's of the $\nu$-th kind $(\nu=1,2)$.

Theorem 2.9. For $\nu=1,2$, there exists a bijection $\Phi^{(\nu)}$ of $\mathscr{G}^{(\nu)}$ to $\mathscr{P}^{(\nu)}$.

Proof. We define the map $\Phi^{(\nu)}$ to be the map $\Phi_{\alpha_{0}}$ in (2.17). Choose an element $\left[\left(\mathfrak{g}_{k}\right)\right] \in \mathscr{G}^{(\nu)}$, and let $\Pi=\Pi_{0} \cup \Pi_{1}$ be the characteristic of $\left(\mathfrak{g}_{k}\right)$ (cf. Lemma 2.8). By a property of the dominant root, we see $\vartheta \in \Delta_{\nu}$. Therefore $\langle\vartheta, E\rangle=\sum_{i=1}^{l} m_{i}(\vartheta)=\nu$, which implies that the cardinality of $\Pi_{1}$ is less than or equal to $\nu$. So the theorem is a direct consequence of Theorem 2.7.

2.3. Let $\left[\Pi_{1}\right] \in \mathscr{P}^{(\nu)}, \nu=1,2$ and put $\Pi_{0}=\Pi-\Pi_{1}$. For a partition $\left(\Pi_{0}, \Pi_{1}\right)$ of $\Pi$, the function $h_{I I}$ in (1.21) is given by $h_{\Pi}(\alpha)=\sum_{\alpha_{i} \in \Pi_{1}} m_{i}(\alpha)$ for $\alpha \in \Delta$. Let us put $\Delta_{k}=\left\{\alpha \in \Delta: h_{I I}(\alpha)=k\right\}, k \in Z$. Then, by Theorem 
2.9 , we have the partition $\Delta=\cup_{k_{k n} \ldots \nu}^{\nu} \Delta_{k}$. In this paragraph we will enumerate this kind of partitions for each irreducible classical root system $\Delta$ which give rise to all gradations of the $\nu$-th kind $(\nu=1,2)$ of classical real simple Lie algebras. We only give $\Delta_{-k}(0 \leqslant k \leqslant \nu)$, since $\Delta_{k}=-\Delta_{-k}$ holds.

1. Type $A_{n-1}(n \geqslant 2)$

$\Delta=\left\{ \pm\left(x_{i}-x_{j}\right): 1 \leqslant i<j \leqslant n\right\}$

$\Pi=\left\{\alpha_{1}, \cdots, \alpha_{n-1}\right\}, \alpha_{i}=x_{i+1}-x_{i}(1 \leqslant i \leqslant n-1)$,

$\vartheta=\alpha_{1}+\cdots+\alpha_{n-1}$.

a) The sets $\left\{\alpha_{p}\right\}(1 \leqslant p \leqslant[n / 2])$ are complete representatives of $\mathscr{P}^{(1)}$. The partition of $\Delta$ for $\Pi_{1}=\left\{\alpha_{p}\right\}$ is given by

$$
\begin{aligned}
& \Delta_{0}=\left\{ \pm\left(x_{i}-x_{j}\right): 1 \leqslant i<j \leqslant p \text { or } p+1 \leqslant i<j \leqslant n\right\} \\
& \Delta_{-1}=\left\{x_{i}-x_{j}: 1 \leqslant i \leqslant p, p+1 \leqslant j \leqslant n\right\} .
\end{aligned}
$$

b) The sets $\left\{\alpha_{p}, \alpha_{p+q}\right\} \quad(1 \leqslant p \leqslant[n / 2], 1 \leqslant q \leqslant n-2 p)$ are complete repesentatives of $\mathscr{P}^{(2)}$. The partition of $\Delta$ for $\Pi_{1}:=\left\{\alpha_{p}, \alpha_{p+q}\right\}$ is given by

$$
\begin{aligned}
& \Delta_{0}=\left\{ \pm\left(x_{i}-x_{j}\right): 1 \leqslant i<j \leqslant p \text { or } p+1 \leqslant i<j \leqslant p+q\right. \\
& \text { or } p+q+1 \leqslant i<j \leqslant n\}, \\
& \Delta_{-1}=\left\{x_{i}-x_{j}: 1 \leqslant i \leqslant p, p+1 \leqslant j \leqslant p+q\right. \\
& \text { or } p+1 \leqslant i \leqslant p+q, p+q+1 \leqslant j \leqslant n\}, \\
& \Delta_{-2}=\left\{x_{i}-x_{j}: 1 \leqslant i \leqslant p, p+q+1 \leqslant j \leqslant n\right\} .
\end{aligned}
$$

2. Type $B_{n}(n \geqslant 2)$

$\Delta=\left\{ \pm\left(x_{i} \pm x_{j}\right)(1 \leqslant i<j \leqslant n), \pm x_{i}(1 \leqslant i \leqslant n)\right\}$,

$\Pi=\left\{\alpha_{1}, \cdots, \alpha_{n}\right\}, \alpha_{i}=x_{i+1}-x_{i}(1 \leqslant i \leqslant n-1), \alpha_{n}=-x_{n}$,

$\vartheta=\alpha_{1}+2\left(\alpha_{2}+\cdots+\alpha_{n}\right)$.

The automorphism group of $\Pi$ is trivial in this case.

c) $\mathscr{P}^{(1)}$ consists of a single set $\left\{\alpha_{1}\right\}$. The corresponding partition of $\Delta$ is given by

$$
\begin{aligned}
& \Delta_{0}=\left\{ \pm\left(x_{i} \pm x_{j}\right)(2 \leqslant i<j \leqslant n), \pm x_{i}(2 \leqslant i \leqslant n)\right\} \\
& \Delta_{-1}=\left\{x_{1} \pm x_{j}(2 \leqslant j \leqslant n), x_{1}\right\} .
\end{aligned}
$$

d) $\mathscr{P}^{(2)}$ consists of the sets $\left\{\alpha_{2}\right\}, \cdots,\left\{\alpha_{n}\right\}$. The partition of $\Delta$ for $\Pi_{1}$ $=\left\{\alpha_{k}\right\}(2 \leqslant k \leqslant n)$ is given by

$$
\begin{aligned}
\Delta_{0}= & \left\{ \pm\left(x_{i}-x_{j}\right)(1 \leqslant i<j \leqslant k), \pm\left(x_{i} \pm x_{j}\right)(k+1 \leqslant i<j \leqslant n),\right. \\
& \left. \pm x_{i}(k+1 \leqslant i \leqslant n)\right\},
\end{aligned}
$$




$$
\begin{aligned}
& \Delta_{-1}=\left\{x_{i} \pm x_{j}(1 \leqslant i \leqslant k, k+1 \leqslant j \leqslant n), x_{i}(1 \leqslant i \leqslant k)\right\}, \\
& \Delta_{-2}=\left\{x_{i}+x_{j}(1 \leqslant i<j \leqslant k)\right\} .
\end{aligned}
$$

3. Type $C_{n}(n \geqslant 3)$

$\Delta=\left\{ \pm\left(x_{i} \pm x_{j}\right)(1 \leqslant i<j \leqslant n), \pm 2 x_{i}(1 \leqslant i \leqslant n)\right\}$,

$\Pi=\left\{\alpha_{1}, \cdots, \alpha_{n}\right\}, \alpha_{i}=x_{i+1}-x_{i}(1 \leqslant i \leqslant n-1), \alpha_{n}=-2 x_{n}$,

$\vartheta=2\left(\alpha_{1}+\cdots+\alpha_{n-1}\right)+\alpha_{n}$.

The automorphism group of $\Pi$ is trivial in this case.

e) $\mathscr{P}^{(1)}$ consists of a single set $\left\{\alpha_{n}\right\}$. The corresponding partition of $\Delta$ is given by

$$
\begin{aligned}
& \Delta_{0}=\left\{ \pm\left(x_{i}-x_{j}\right): 1 \leqslant i<j \leqslant n\right\}, \\
& \Delta_{-1}=\left\{x_{i}+x_{j}(1 \leqslant i<j \leqslant n), 2 x_{i}(1 \leqslant i \leqslant n)\right\} .
\end{aligned}
$$

f) $\mathscr{P}^{(2)}$ consists of the sets $\left\{\alpha_{1}\right\}, \cdots,\left\{\alpha_{n-1}\right\}$. The partition of $\Delta$ for $\Pi_{1}=\left\{\alpha_{k}\right\}(1 \leqslant k \leqslant n-1)$ is given by

$$
\begin{aligned}
\Delta_{0}= & \left\{ \pm\left(x_{i}-x_{j}\right)(1 \leqslant i<j \leqslant k), \pm\left(x_{i} \pm x_{j}\right)(k+1 \leqslant i<j \leqslant n),\right. \\
& \left. \pm 2 x_{i}(k+1 \leqslant i \leqslant n)\right\}, \\
\Delta_{-1}= & \left\{x_{i} \pm x_{j}(1 \leqslant i \leqslant k, k+1 \leqslant j \leqslant n)\right\}, \\
\Delta_{-2}= & \left\{x_{i}+x_{j}(1 \leqslant i<j \leqslant k), 2 x_{i}(1 \leqslant i \leqslant k)\right\} .
\end{aligned}
$$

4. Type $B C_{n}(n \geqslant 1)$

$\Delta=\left\{ \pm\left(x_{i} \pm x_{j}\right)(1 \leqslant i<j \leqslant n), \pm x_{i}, \pm 2 x_{i}(1 \leqslant i \leqslant n)\right\}$,

$\Pi=\left\{\alpha_{1}, \cdots, \alpha_{n}\right\}, \alpha_{i}=x_{i+1}-x_{i}(1 \leqslant i \leqslant n-1), \alpha_{n}=-x_{n}$,

$\vartheta=2 \alpha_{1}+\cdots+2 \alpha_{n}$.

The automorphism group of $\Pi$ is trivial in this case. $\mathscr{P}^{(1)}$ is empty.

g) $\mathscr{P}^{(2)}$ consists of the sets $\left\{\alpha_{1}\right\}, \cdots,\left\{\alpha_{n}\right\}$. The partition of $\Delta$ for $\Pi_{1}$ $=\left\{\alpha_{k}\right\}(1 \leqslant k \leqslant n)$ is given by

$$
\begin{aligned}
\Delta_{0}= & \left\{ \pm\left(x_{i}-x_{j}\right)(1 \leqslant i<j \leqslant k), \pm\left(x_{i} \pm x_{j}\right)(k+1 \leqslant i<j \leqslant n),\right. \\
& \pm x_{i}, \pm 2 x_{i}(k+1 \leqslant i \leqslant n), \\
\Delta_{-1}= & \left\{x_{i} \pm x_{j}(1 \leqslant i \leqslant k, k+1 \leqslant j \leqslant n), x_{i}(1 \leqslant i \leqslant k)\right\} \\
\Delta_{-2}= & \left\{x_{i}+x_{j}(1 \leqslant i<j \leqslant k), 2 x_{i}(1 \leqslant i \leqslant k)\right\} .
\end{aligned}
$$

5. Type $D_{n}(n \geqslant 4)$

$\Delta=\left\{ \pm\left(x_{i} \pm x_{j}\right): 1 \leqslant i<j \leqslant n\right\}$,

$\Pi=\left\{\alpha_{1}, \cdots, \alpha_{n}\right\}, \alpha_{i}=x_{i+1}-x_{i}(1 \leqslant i \leqslant n-1), \alpha_{n}=-x_{n-1}-x_{n}$,

$\vartheta=\alpha_{1}+2\left(\alpha_{2}+\cdots+\alpha_{n-2}\right)+\alpha_{n-1}+\alpha_{n}$.

The sets $\left\{\alpha_{1}\right\},\left\{\alpha_{n}\right\}$ are complete representatives of $\mathscr{P}^{(1)}$ for $n \neq 4$, while 
$\left\{\alpha_{1}\right\}$ is for $n=4$. The sets $\left\{\alpha_{2}\right\}, \cdots,\left\{\alpha_{n-2}\right\},\left\{\alpha_{1}, \alpha_{n}\right\},\left\{\alpha_{n-1}, \alpha_{n}\right\}$ are complete representatives of $\mathscr{P}^{(2)}$ for $n \neq 4$, while $\left\{\alpha_{2}\right\},\left\{\alpha_{3}, \alpha_{4}\right\}$ are for $n=4$.

h) The partition of $\Delta$ for $\Pi_{1}=\left\{\alpha_{1}\right\}$ is given by

$$
\Delta_{0}=\left\{ \pm\left(x_{i} \pm x_{j}\right): 2 \leqslant i<j \leqslant n\right\}, \quad \Delta_{-1}=\left\{x_{1} \pm x_{j}: 2 \leqslant j \leqslant n\right\} .
$$

i) The partition of $\Delta$ for $\Pi_{1}=\left\{\alpha_{n}\right\}$ is given by

$$
\Delta_{0}=\left\{ \pm\left(x_{i}-x_{j}\right): 1 \leqslant i<j \leqslant n\right\}, \quad \Delta_{-1}=\left\{x_{i}+x_{j}: 1 \leqslant i<j \leqslant n\right\} .
$$

j) The partition of $\Delta$ for $\Pi_{1}=\left\{\alpha_{k}\right\}(2 \leqslant k \leqslant n-2)$ is given by

$$
\begin{aligned}
& \Delta_{0}=\left\{ \pm\left(x_{i}-x_{j}\right)(1 \leqslant i<j \leqslant k), \pm\left(x_{i} \pm x_{j}\right)(k+1 \leqslant i<j \leqslant n)\right\} \\
& \Delta_{-1}=\left\{x_{i} \pm x_{j}: 1 \leqslant i \leqslant k, k+1 \leqslant j \leqslant n\right\} \\
& \Delta_{-2}=\left\{x_{i}+x_{j}: 1 \leqslant i<j \leqslant k\right\} .
\end{aligned}
$$

k) The partition of $\Delta$ for $\Pi_{1}=\left\{\alpha_{n-1}, \alpha_{n}\right\}$ is given by

$$
\begin{aligned}
& \Delta_{0}=\left\{ \pm\left(x_{i}-x_{j}\right): 1 \leqslant i<j \leqslant n-1\right\}, \\
& \Delta_{-1}=\left\{x_{i} \pm x_{n}: 1 \leqslant i \leqslant n-1\right\}, \\
& \Delta_{-2}=\left\{x_{i}+x_{j}: 1 \leqslant i<j \leqslant n-1\right\} .
\end{aligned}
$$

1) The partition of $\Delta$ for $\Pi_{1}=\left\{\alpha_{1}, \alpha_{n}\right\}$ is given by

$$
\begin{aligned}
& \Delta_{0}=\left\{ \pm\left(x_{i}-x_{j}\right): 2 \leqslant i<j \leqslant n\right\}, \\
& \Delta_{-1}=\left\{x_{1}-x_{j}(2 \leqslant j \leqslant n), x_{i}+x_{j}(2 \leqslant i<j \leqslant n)\right\}, \\
& \Delta_{-2}=\left\{x_{1}+x_{j}(2 \leqslant j \leqslant n)\right\} .
\end{aligned}
$$

Remark 2.10. Let $g$ be a classical real simple Lie algebra, and let $\left(\mathfrak{g}, \Pi_{1}\right.$ ) denote the gradation of the $\nu$-th kind in $\mathfrak{g}$ corresponding to $\Pi_{1}$. By using a)-l) above, we see that $\left(g, \Pi_{1}\right)$ satisfies $\nu=2$ and $\operatorname{dim} g_{-2}=1$, if and only if $\left(\mathfrak{g}, \Pi_{1}\right)$ is one of the followings: $\left(\mathfrak{g l}(n, \boldsymbol{R}),\left\{\alpha_{1}, \alpha_{n-1}\right\}\right)(n \geqslant 3)$, $\left(\mathfrak{I H}(p, q),\left\{\alpha_{1}\right\}\right)(1 \leqslant p \leqslant q),\left(\mathfrak{I D}(p, q),\left\{\alpha_{2}\right\}\right)(2 \leqslant p \leqslant q),\left(\mathfrak{I} \mathfrak{p}(n, \boldsymbol{R}),\left\{\alpha_{1}\right\}\right)(n \geqslant 3)$, $\left(\mathfrak{S O}^{*}(2 n),\left\{\alpha_{1}\right\}\right)(n \geqslant 4)$. This reproduces the Cheng's result stated in Introduction for $g$ classical.

\section{$\S 3$. The Lie algebra $\mathscr{L}(B)$ and GJTS's}

3.1. For the later use we will mention some properties of a universal graded Lie algebra and the GLA $\mathscr{L}(B)$ both due to Kantor [6], [7]. For convenience, we denote by $a_{1} \circ a_{2}$ the commutator product $\left[a_{1}, a_{2}\right]$ in a Lie algebra, and define $a_{1} \circ \cdots \circ a_{m}$ inductively by $\left(a_{1} \circ \cdots \circ a_{m-1}\right) \circ a_{m}$. Let $\mathscr{U}_{-1}$ be a finite-dimensional vector space, and $\mathscr{U}_{-}$be the free Lie algebra 
generated by $\mathscr{U}_{-1}$ [2]. Let $\mathscr{U}_{-m}$ be the subspace of $\mathscr{U}_{-}$which is spanned by elements of the form $a_{1} \circ \ldots \circ a_{m}$, where $a_{1}, \cdots, a_{m} \in \mathscr{U}_{-1}$. Then one can write $\mathscr{U}_{-}$in the form of a GLA:

$$
\mathscr{U}_{-}=\sum_{i=1}^{\infty} \mathscr{U}_{-i}
$$

Let $\mathscr{U}_{-1}^{*}$ be the dual space of $\mathscr{U}_{-1}$ and let

$$
\mathscr{U}_{n}=\left(\otimes{ }^{n+1} \mathscr{U}_{-1}^{*}\right) \otimes \mathscr{U}_{-1},
$$

whose elements are viewed as $\mathscr{U}_{-1}$-valued $(n+1)$-linear operators on $\mathscr{U}_{-1}$. Put

$$
\begin{aligned}
& \mathscr{U}_{+}=\sum_{i=0}^{\infty} \mathscr{U}_{i}, \\
& \mathscr{U}=\mathscr{U}_{-}+\mathscr{U}_{+}=\sum_{i=-\infty}^{\infty} \mathscr{U}_{i} .
\end{aligned}
$$

Then it is known [6] that, with respect to suitably defined bracket relations, $\mathscr{U}$ becomes a GLA in which $\mathscr{U}_{-}$is a graded subalgebra. The GLA is called the universal graded Lie algebra (or simply UGLA) generated by $\mathscr{U}_{-1}$. The assignment $\mathscr{U}_{-1} \mapsto \mathscr{U}$ has a functorial property in the following sense: Let $\varphi$ be a linear isomorphism of $\mathscr{U}_{-1}$ onto another vector space $\mathscr{V}_{-1}$. Then it is easy to see that $\varphi$ naturally extends to a gradepreserving Lie isomorphism $\hat{\varphi}$ of the UGLA $\mathscr{U}$ generated by $\mathscr{U}_{-1}$ onto the UGLA $\mathscr{V}$ generated by $\mathscr{V}_{-1}$. Here $\left.\hat{\varphi}\right|_{\mathscr{\psi}_{-}}$is the mapping, induced by $\varphi$, between the free Lie algebras $\mathscr{U}_{-}$and $\mathscr{V}_{-} ;\left.\hat{\varphi}\right|_{\mathscr{U}_{+}}$comes from the mapping, induced by $\varphi$, between the tensor algebras over $\mathscr{U}_{-1}$ and over $\mathscr{V}_{-1}$.

Definition 3.1. A GLA $U=\sum_{i=-\infty}^{\infty} U_{i}$ is said to be of type $\alpha$ if it is of type $\alpha_{0}$ and if $U_{+}=\sum_{i \geqslant 0} U_{i}$ contains no ideal of $U$ expect (0) and $\sum_{i \geqslant 2} U_{-i}$ contains no graded ideal of $U$ other than (0).

Definition 3.2. Let $V=\sum_{i=-\infty}^{\infty} V_{i}$ be a GLA. The subspace $V_{-1}+$ $V_{0}+V_{1}$ is called the local part of $V$ and is denoted by loc $(V)$. Let $U=$ $\sum_{i=-\infty}^{\infty} U_{i}$ be another GLA. A linear map $\varphi$ of $\operatorname{loc}(V)$ into $\operatorname{loc}(U)$ is called a homomorphism between the local parts, if it satisfies

$$
\varphi\left(\left[x_{i}, x_{j}\right]\right)=\left[\varphi\left(x_{i}\right), \varphi\left(x_{j}\right)\right] \quad x_{i} \in V_{i}, x_{j} \in V_{j},
$$

where $(i, j)=(0,0),(-1,0),(-1,1)$ and $(0,1)$. Moreover, if $\varphi$ is bijective, then it is called an isomorphism. 
3.2. Let $U_{-1}$ be a (finite-dimensional) vector space and $\mathrm{B}: U_{-1} \times$ $U_{-1} \times U_{-1} \rightarrow U_{-1}$ be a trilinear mapping. Then the pair $\left(U_{-1}, B\right)$ (sometimes denoted by $B$ for brevity) is called a triple system. We shall often write $(x y z)$ instead of $B(x, y, z)$. A triple system $\left(U_{-1}, B\right)$ is called a generalized Jordan triple system (or shortly GJTS), if the equality

$$
(u v(x y z))=((u v x) y z)-(x(v u y) z)+(x y(u v z))
$$

is valid for $u, v, x, y, z \in U_{-1}$. Furthermore, if the additional condition

$$
(x y z)=(z y x) \quad x, y, z \in U_{-1}
$$

is satisfied, then $B$ is called a Jordan triple system (or simply JTS).

Definition 3.3. Let $\left(U_{-1}, B\right)$ and $\left(V_{-1}, B^{\prime}\right)$ be two GJTS's. We say that a linear map $\varphi$ of $U_{-1}$ into $V_{-1}$ is a homomorphism if $\varphi$ satisfies

$$
\varphi(B(x, y, z))=B^{\prime}(\varphi(x), \varphi(y), \varphi(z)) \quad x, y, z \in U_{-1} .
$$

Moreover, if $\varphi$ is bijective, then $\varphi$ is called an isomorphism. In this case, $\left(U_{-1}, B\right)$ and $\left(V_{-1}, B^{\prime}\right)$ are said to be isomorphic.

Definition 3.4. Let $\left(U_{-1}, B\right)$ be a GJTS. A subspace $V$ of $U_{-1}$ is called an ideal (resp. K-ideal) if

$$
\begin{gathered}
B\left(V, U_{-1}, U_{-1}\right)+B\left(U_{-1}, V, U_{-1}\right)+B\left(U_{-1}, U_{-1}, V\right) \subset V \\
\left(\text { resp. } B\left(V, U_{-1}, U_{-1}\right)+B\left(U_{-1}, U_{-1}, V\right) \subset V\right)
\end{gathered}
$$

is valid. $\left(U_{-1}, B\right)$ is called simple (resp. $K$-simple), if $B$ is not a zero map and if $\left(U_{-1}, B\right)$ has no non-trivial ideal (resp. $K$-ideal). We say that $\left(U_{-1}, B\right)$ satisfies the condition $(A)$ if $B\left(U_{-1}, a, U_{-1}\right)=0$ implies $a=0$.

Obviously $K$-simplicity implies simplicity, but the converse is not always true (cf. [8], [1]). It is known [1] that simplicity implies the condition $(A)$.

Now let $\left(U_{-1}, B\right)$ be a GJTS and let $\mathscr{U}=\sum_{i=-\infty}^{\infty} \mathscr{U}_{i}$ be the UGLA generated by $\mathscr{U}_{-1}:=U_{-1}$. We put

$$
\begin{aligned}
& L_{a b}(x)=B(a, b, x)=(a b x), \\
& R_{a b}(x)=B(x, a, b)=(x a b), \\
& B_{a}(x, y)=B(x, a, y)=(x a y) .
\end{aligned}
$$

Note that $L_{a b}, R_{a b} \in \mathscr{U}_{0}$ and $B_{a} \in \mathscr{U}_{1}$. Let $U_{1}$ be the subspace of $\mathscr{U}_{1}$ consisting of all operators $B_{a}, a \in U_{-1}$, and let $U_{0}$ be the subspace of $\mathscr{U}_{0}$ 
spanned by operators $L_{a b}$, where $a, b \in U_{-1}$. In the UGLA $\mathscr{U}$ we have

$$
\begin{aligned}
& {\left[B_{a}, b\right]=L_{b a} \quad a, b \in U_{-1},} \\
& {\left[L_{a b}, B_{c}\right]=-B_{(b a c)},} \\
& {\left[L_{a b}, L_{c d}\right]=L_{(a b c) d}-L_{c(b a d)} .}
\end{aligned}
$$

Hence we get

$$
\left[U_{-1}, U_{1}\right]=U_{0}, \quad\left[U_{0}, U_{0}\right] \subset U_{0}, \quad\left[U_{0}, U_{1}\right] \subset U_{1} .
$$

Let $\mathscr{L}_{0}(B)$ be the (graded) subalgebra of $\mathscr{U}$ generated by the subspaces $U_{-1}$ and $U_{1}$. $\mathscr{L}_{0}(B)$ can be written as

$$
\mathscr{L}_{0}(B)=\mathscr{U}_{-}+\sum_{i \geqslant 0} U_{i} .
$$

It is of type $\alpha_{0}$. Furthermore it can be seen (Lemma 5 [6]) that $\sum_{i \geqslant 0} U_{i}$ contains no ideal of $\mathscr{L}_{0}(B)$ other than zero. Let $D$ be a maximal graded ideal of $\mathscr{L}_{0}(B)$ contained in $\sum_{i \geqslant 2} \mathscr{U}_{-i}$. Note that such an ideal $D$ is unique. We define the GLA $\mathscr{L}(B)=\sum_{i=-\infty}^{\infty} V_{i}$ to be

$$
\mathscr{L}(B)=\mathscr{L}_{0}(B) / D,
$$

which is of type $\alpha$ (not necessarily of finite dimension) [7]. $\mathscr{L}(B)$ is uniquely determined by the given GJTS $\left(U_{-1}, B\right)$. We call $\mathscr{L}(B)$ the Kantor algebra for $B$. By the definition, the subalgebra $\mathscr{L}(B)_{+}=\sum_{i \geqslant 0} V_{i}$ is canonically isomorphic to $\mathscr{L}_{0}(B)_{+}=\sum_{i \geqslant 0} U_{i}$. So, in the sequel, we will regard $\mathscr{L}(B)_{+}$as a subalgebra of $\mathscr{U}$ via the above isomorphism. We need the following

Theorem 3.5 ([7], [6]). (i) Let $\left(U_{-1}, B\right)$ be a GJTS. If $\left(U_{-1}, B\right)$ is $K$-simple and if $\operatorname{dim} \mathscr{L}(B)<\infty$, then the Lie algebra $\mathscr{L}(B)$ is simple. (ii) Conversely, let $V=\sum_{i=-\infty}^{\infty} V_{i}$ be a simple GLA of type $\alpha_{0}$ and let $\tau$ be a grade-reversing involutive automorphism of $V$. If we define a trilinear $\operatorname{map} B_{\tau}$ by

$$
B_{\tau}(y, x, z)=[[\tau(x), y], z], \quad x, y, z \in V_{-1},
$$

then $\left(V_{-1}, B_{\tau}\right)$ is a K-simple GJTS.

3.3. In this paragraph, we construct a grade-reversing involutive automorphism $\tau_{B}$ of $\mathscr{L}(B)$. This was done originally by Kantor [7], but the proof given there is rather sketchy; so we give it rigorously by relaxing the condition "center-free" to the condition (A). Let $\left(U_{-1}, B\right)$ be a GJTS 
satisfying the condition (A), and let $\mathscr{L}(B)=\sum_{i=-\infty}^{\infty} U_{i}$. Consider the mapping $\tau: U_{-1} \rightarrow U_{1}$ :

$$
\tau(a)=B_{a}
$$

The condition (A) implies that $\tau$ is a linear isomorphism of $U_{-1}$ onto $U_{1}$. Let $\mathscr{L}^{\prime}(B)=\sum_{i=-\infty}^{\infty} V_{i}$ be the GLA which is obtained from $\mathscr{L}(B)$ by reversing the gradation, that is, by putting $V_{i}=U_{-i}$ for each $i$. Let $\mathscr{U}=$ $\sum_{i=-\infty}^{\infty} \mathscr{U}_{i}$ and $\mathscr{V}=\sum_{i=-\infty}^{\infty} \mathscr{V}_{i}$ be the UGLA's generated by $\mathscr{U}_{-1}=U_{-1}$ and $\mathscr{V}_{-1}=V_{-1}=U_{1}$, respectively. Let us consider the two subalgebras $\mathscr{L}(B)^{(1)}=\sum_{i \geqslant 1} U_{i} \subset \mathscr{L}(B)$ and $\mathscr{L}^{\prime}(B)^{(1)}=\sum_{i \geqslant 1} V_{i} . \quad \mathscr{L}(B)^{(1)}$ is viewed as a graded subalgebra of $\mathscr{U}$ (cf. the statement just before Theorem 3.5). Let $a \in V_{i}(i \geqslant 1)$ and let

$$
F(a)\left(x_{1}, \cdots, x_{i+1}\right)=a \circ x_{1} \circ \cdots \circ x_{i+1},
$$

where $x_{1}, \cdots, x_{i+1} \in V_{-1}=\mathscr{V}_{-1}$. Then $F(a)$ is a $V_{-1}$-valued $(i+1)$-linear form on $V_{-1}$, and hence $F(a) \in \mathscr{V}_{i}$. We extend $F$ linearly to the whole $\mathscr{L}^{\prime}(B)^{(1)}$.

Lemma 3.6. The mapping $F$ is an injective grade-preserving homomorphism of $\mathscr{L}^{\prime}(B)^{(1)}$ into $\mathscr{V}_{+}=\sum_{i \geqslant 0} \mathscr{V}_{i}$.

Proof. It is known [6] that $F$ is a grade-preserving homomorphism. We claim first that $\left.F\right|_{V_{1}}$ is injective. Let $a \in V_{1}$ and suppose that $F(a)=0$. Then, from (3.10), (3.11), and (3.18), we have

$$
0=F(a)(\tau(u), \tau(v))=\left[\left[a, B_{u}\right], B_{v}\right]=B_{(u a v)}=\tau((u a v))
$$

for $\tau(u), \tau(v) \in \mathscr{V}_{-1}=V_{-1}=U_{1}$. Since $\tau: U_{-1} \rightarrow U_{1}$ is a linear isomorphism, we have that $(u a v)=0$ for all $u, v \in U_{-1}$, and hence, by the condition (A), $a=0$. Therefore Ker $F$ is contained in $\sum_{i \geqslant 2} V_{i}$. It can be seen (cf. the proof of Lemma 4 [6]) that $\operatorname{Ker} F$ is an ideal of the whole $\mathscr{L}^{\prime}(B)$, that is, Ker $F$ is an ideal of $\mathscr{L}(B)$ contained in $\sum_{i \leqslant-2} U_{i}$. Since $\mathscr{L}(B)$ is of type $\alpha$, we have $\operatorname{Ker} F=(0)$.

By Lemma 3.6, we may identify $\mathscr{L}^{\prime}(B)^{(1)}$ with its $F$-image in $\mathscr{V}_{+}$. The linear isomorphism $\tau$ of $\mathscr{U}_{-1}$ onto $\mathscr{V}_{-1}$ naturally extends to a grade-preserving isomorphism $\hat{\tau}$ of $\mathscr{U}$ onto $\mathscr{V}$ (cf. 3.1).

Lemma 3.7. $\left.\hat{\tau}\right|_{U_{1}}=\tau^{-1}$ is valid. Furthermore $\hat{\tau}$ sends $\mathscr{L}(B)^{(1)}$ to $\mathscr{L}^{\prime}(B)^{(1)}$. 
Proof. Choose $B_{a} \in U_{1} \subset \mathscr{U}_{1}\left(a \in U_{-1}\right)$ and let $\hat{\tau}\left(B_{a}\right)=B^{\prime} \in \mathscr{V}_{1}$. Then, by the naturality of $\hat{\tau}$ we have

$$
B^{\prime}(\tau(u), \tau(v))=\tau\left(B_{a}(u, v)\right) \quad u, v \in U_{-1},
$$

from which it follows that

$$
B^{\prime}\left(B_{u}, B_{v}\right)=\tau((u a v))=B_{(u a v)}=F(a)\left(B_{u}, B_{v}\right)=a\left(B_{u}, B_{v}\right) .
$$

$B_{u}$ and $B_{v}$ being arbitrary, we get $\hat{\tau}\left(B_{a}\right)=B^{\prime}=a$, which implies that $\left.\hat{\tau}\right|_{U_{1}}=\tau^{-1}$. Hence $\hat{\tau}\left(U_{1}\right)=\tau^{-1}\left(U_{1}\right)=U_{-1}=V_{1}$. Since $\mathscr{L}(B)$ is of type $\alpha$, the subalgebra $\mathscr{L}(B)^{(1)}$ (resp. $\left.\mathscr{L}^{\prime}(B)^{(1)}\right)$ is generated by $U_{1}$ (resp. $V_{1}$ ) in $\mathscr{U}$ (resp. $\mathscr{V})$. Therefore we conclude that $\hat{\tau}$ sends $\mathscr{L}(B)^{(1)}$ to $\mathscr{L}^{\prime}(B)^{(1)}$.

Now we define $\tau_{B}$ as

$$
\tau_{B}= \begin{cases}\hat{\tau} & \text { on } \mathscr{L}(B)^{(1)}, \\ \hat{\tau}^{-1} & \text { on } \mathscr{L}(B)_{-}:=\sum_{i \leqslant-1} U_{i}=\mathscr{L}^{\prime}(B)^{(1)} .\end{cases}
$$

From Lemma 3.7, we have that $\tau_{B}$ is an involutive linear endomorphism of $\mathscr{L}(B)_{-}+\mathscr{L}(B)^{(1)}$ and that $\tau_{B}$ is a Lie homomorphism both on $\mathscr{L}(B)^{(1)}$ and on $\mathscr{L}(B)_{\text {.. }}$. We extend $\tau_{B}$ to an involutive linear endomorphism (denoted again by $\tau_{B}$ ) of the whole $\mathscr{L}(B)$ by putting

$$
\tau_{B}\left(L_{a b}\right)=-L_{b a} \quad L_{a b} \in U_{0} .
$$

The following proposition is a variation of Proposition $6^{\prime}$ in Kantor [7], in which we relax an assumption in the original one.

Proposition 3.8. Let $\left(U_{-1}, B\right)$ be a GJTS satisfying the condition (A). Then the linear endomorphism $\tau_{B}$ defined by (3.22) and (3.23) is a gradereversing involutive automorphism of $\mathscr{L}(B)$.

Proof. By using (3.22) and (3.23), and by following Kantor [7] (p. 428 ), we can show that $\tau_{B}$ is a homomorphism.

$\tau_{B}$ is called the grade-reversing canonical involution of $\mathscr{L}(B)$.

3.4. Let $\left(U_{-1}, B\right)$ be a GJTS satisfying the condition (A). If the GLA $\mathscr{L}(B)$ is of the $\nu$-th kind, then we say that $\left(U_{-1}, B\right)$ is of the $\nu$-th kind. Note that $B$ is a JTS if and only if $\nu=1$ [7]. The symmetric bilinear form $\gamma_{B}$ [19] on $U_{-1}$ defined by

$$
\gamma_{B}(x, y)=\frac{1}{2} \operatorname{Tr}\left(2 R_{x y}+2 R_{y x}-L_{x y}-L_{y x}\right)
$$

is called the trace form of the GJTS $\left(U_{-1}, B\right)$. If $\gamma_{B}$ is positive definite, 
then $\left(U_{-1}, B\right)$ is said to be compact. When $\nu=1$, our definition of "compactness" is the same as that for JTS's. Suppose that $\left(U_{-1}, B\right)$ is a GJTS of the first or the second kind satisfying the condition (A). Then it is known [1] that $\left(U_{-1}, B\right)$ is compact if and only if $\mathscr{L}(B)$ is semisimple and $\tau_{B}$ is a Cartan involution. Suppose that $\left(U_{-1}, B\right)$ is compact of the first or the second kind. Then $\left(U_{-1}, B\right)$ is simple if and only if it is $K$ simple ([1]).

3.5. In this paragraph we treat infinite-dimensional simple GLA's. Let $\mathfrak{g}=\sum_{i=-\infty}^{\infty} \mathfrak{g}_{i}$ be a simple GLA of type $\alpha_{0}$ and $\tau$ be a grade-reversing involutive automorphism of $\mathfrak{g}$. Then $\left(\mathfrak{g}_{-1}, B_{z}\right)$ is a $K$-simple GJTS (cf. Theorem 3.5), and hence it satisfies the condition (A) (cf. 3.2). Therefore the Kantor algebra $\mathscr{L}\left(B_{\imath}\right)$ admits the grade-reversing canonical involution $\tau_{B_{\tau^{*}}}$ Consider the UGLA $\mathscr{U}=\sum_{i=-\infty}^{\infty} \mathscr{U}_{i}$ generated by $\mathscr{U}_{-1}=\mathfrak{g}_{-1}$ and consider the subalgebra $\mathscr{L}_{0}\left(B_{\tau}\right)$ of $\mathscr{U}$ given in (3.14). We then have

LEMMA 3.9. $\operatorname{loc}(\mathfrak{g}) \cong \operatorname{loc}\left(\mathscr{L}_{0}\left(B_{\tau}\right)\right)$.

Proof. Let $\mathscr{L}_{0}\left(B_{\tau}\right)=\mathscr{U}_{-}+\sum_{i \geqslant 0} U_{i}$. Let $\varphi_{-1}$ be the identity map of $\mathfrak{g}_{-1}$ onto $\mathscr{U}_{-1}$. We define $\varphi_{1}: \mathfrak{g}_{1} \rightarrow U_{1}$ be $\varphi_{1}(\tau(u))=\left(B_{z}\right)_{u}$ for $u \in \mathfrak{g}_{-1}$. Then, since $\tau_{B_{\tau}}(u)=\left(B_{\tau}\right)_{u}$ (cf. (3.22)), we have $\varphi_{1}=\tau_{B_{\tau}} \tau^{-1} . \quad \tau_{B_{\tau}}$ is a bijection of $\mathfrak{g}_{-1}=\mathscr{U}_{-1}$ to $U_{1}$, and so $\varphi_{1}$ is a linear isomorphism of $\mathfrak{g}_{1}$ onto $U_{1}$. Since $\mathfrak{g}$ is simple, we easily see $\left[\mathfrak{g}_{-1}, \mathfrak{g}_{1}\right]=\mathfrak{g}_{0}$. We define $\varphi_{0}: \mathfrak{g}_{0} \rightarrow U_{0}$ by putting $\varphi_{0}([\tau(u), v])=L_{v u}\left(u, v \in \mathfrak{g}_{-1}\right)$. We claim that $\varphi_{0}$ is a bijection. First note that the representation $\rho: \mathfrak{g}_{0} \rightarrow \operatorname{ad}_{\mathfrak{g}-1} \mathfrak{g}_{0}$ is faithful. Indeed, suppose the contrary; choose a non-zero element $x \in \mathfrak{g}_{0}$ such that $\left[x, \mathfrak{g}_{-1}\right]=0$. Then $\sum_{k, l \geqslant 0}\left(\operatorname{ad~} g_{1}\right)^{k}\left(\operatorname{ad~g}_{0}\right)^{l} x$ is a non-zero ideal of $\mathrm{g}$. But this is impossible, since $\mathrm{g}$ is simple. That $\varphi_{0}$ is surjective is trivial. Choose an element $a=$ $\sum_{i=1}^{s} \lambda_{i}\left[\tau\left(u_{i}\right), v_{i}\right] \in \mathfrak{g}_{0}\left(\lambda_{i} \in R\right)$, and suppose $\varphi_{0}(a)=0$. Then, for every $x \in \mathfrak{g}_{-1}$ $=\mathscr{U}_{-1}$, we have $[a, x]=\sum_{i} \lambda_{i}\left[\left[\tau\left(u_{i}\right), v_{i}\right], x\right]=\sum_{i} \lambda_{i} B_{\tau}\left(v_{i}, u_{i}, x\right)=\sum_{i} \lambda_{i} L_{v_{i} u_{i}}(x)$ $=\varphi_{0}(a) x=0$. Since $\rho$ is faithful, we get $a=0$. Thus $\varphi_{0}$ is a bijection. We shall prove that the linear bijection $\varphi=\varphi_{-1} \times \varphi_{0} \times \varphi_{1}$ is an isomorphism of $\operatorname{loc}(\mathrm{g})$ onto $\operatorname{loc}\left(\mathscr{L}_{0}\left(B_{z}\right)\right)$. Let $x \in \mathfrak{g}_{-1}$ and $y=[\tau(u), v] \in \mathfrak{g}_{0}$, where $u, v \in$ $g_{-1}$. Then we have

$$
\begin{aligned}
\varphi_{-1}([y, x]) & =[y, x]=[[\tau(u), v], x]=B_{\tau}(v, u, x)=L_{v u}(x) \\
& =\left[L_{v u}, x\right]=\left[\varphi_{0}(y), \varphi_{-1}(x)\right] .
\end{aligned}
$$

Let $u, v \in \mathfrak{g}_{-1}$. Then, for $x \in \mathfrak{g}_{-1}=\mathscr{U}_{-1}$ we get 


$$
\begin{aligned}
{\left[\varphi_{1}(\tau(u)), \varphi_{-1}(v)\right](x) } & =\left[\left(B_{\tau}\right)_{u}, v\right](x)=B_{\tau}(v, u, x)=L_{v u}(x) \\
& =\varphi_{0}([\tau(u), v])(x) .
\end{aligned}
$$

Let $w \in \mathfrak{g}_{-1}$. Then we have

$$
\begin{aligned}
\varphi_{1}([y, \tau(w)]) & =\varphi_{1}([[\tau(u), v], \tau(w)])=\varphi_{1}(\tau([[u, \tau(v)], w])) \\
& =\left(B_{\tau}\right)_{[[u, \tau(v)], w]} .
\end{aligned}
$$

On the other hand, by using (3.11), we get

$$
\begin{aligned}
{\left[\varphi_{0}(y), \varphi_{1}(\tau(w))\right] } & =\left[\varphi_{0}([\tau(u), v]), \varphi_{1}(\tau(w))\right]=\left[L_{v u},\left(B_{\tau}\right)_{w}\right] \\
& =-\left(B_{\tau}\right)_{(u v w)} .
\end{aligned}
$$

Furthermore $-(u v w)=-B_{\tau}(u, v, w)=[[u, \tau(v)], w]$. Thus we have proved $\varphi_{1}([y, \tau(w)])=\left[\varphi_{0}(y), \varphi_{1}(\tau(w))\right]$. Using the above equalities, one can show that $\varphi_{0}([x, y])=\left[\varphi_{0}(x), \varphi_{0}(y)\right]$ for $x, y \in \mathfrak{g}_{0}$.

Lemma 3.10. Let $\mathfrak{g}=\sum_{i=-\infty}^{\infty} \mathfrak{g}_{i}$ be a real simple GLA of type $\alpha_{0}$. Let $\tau$ be a grade-reversing involutive automorphism of $\mathrm{g}$, and let $\mathscr{L}\left(B_{\tau}\right)$ be the Kantor algebra for the GJTS $\left(\mathfrak{g}_{-1}, B_{\tau}\right)$. Then there exists a grade-preserving isomorphism $\varphi$ of $\mathfrak{g}$ onto $\mathscr{L}\left(B_{\tau}\right)$ such that

$$
\varphi \tau=\tau_{B_{\tau}} \varphi \text {. }
$$

Proof. Let $\mathscr{U}=\sum_{i=-\infty}^{\infty} \mathscr{U}_{i}$ be the UGLA such that $\mathscr{U}_{-1}=\mathfrak{g}_{-1}$. For a GLA $\mathfrak{h}=\sum_{i=-\infty}^{\infty} \mathfrak{h}_{i}$, we put $\mathfrak{h}_{+}=\sum_{i \geqslant 0} \mathfrak{h}_{i}$ and $\mathfrak{h}_{-}=\sum_{i \leqslant-1} \mathfrak{h}_{i}$. We define a map $F$ of $\mathfrak{g}_{+}$to $\mathscr{U}_{+}$quite analogously as in (3.18). Note that (3.18) is meaningful for $i=0$. Since $g$ is simple of type $\alpha_{0}$, the map $F$ is an injective grade-preserving homomorphism [6]. An easy computation shows that $F$ coincides with $\varphi_{0} \times \varphi_{1}$ on $\mathfrak{g}_{0}+\mathfrak{g}_{1}$. Let $\mathscr{L}\left(B_{\tau}\right)=\sum_{i=-\infty}^{\infty} U_{i}$. We identify $\mathscr{L}\left(B_{\tau}\right)_{+}$with $\mathscr{L}_{0}\left(B_{\tau}\right)_{+} \subset \mathscr{U}_{+}$. Then, as is seen in the proof of Lemma 3.9 , we have $F\left(\mathfrak{g}_{0}+\mathfrak{g}_{1}\right)=U_{0}+U_{1}$. Considering that $g$ and $\mathscr{L}\left(B_{\tau}\right)$ are both of type $\alpha_{0}$, we conclude that the GLA's $\mathfrak{g}_{+}$and $\mathscr{L}\left(B_{\tau}\right)_{+}$are isomorphic under $F$. The map $F^{\prime}:=\tau_{B_{\tau}} F_{\tau}$ is a grade-preserving isomorphism of $g_{-}$onto $\mathscr{L}\left(B_{\tau}\right)_{-}$. We define a map $\varphi$ to be $F$ on $\mathfrak{g}_{+}$and to be $F^{\prime}$ on $\mathrm{g}_{-}$. Then $\varphi$ is a grade-preserving linear isomorphism of $\mathfrak{g}$ onto $\mathscr{L}\left(B_{\imath}\right)$. $\varphi$ is an isomorphism between $\operatorname{loc}(\mathrm{g})$ and $\operatorname{loc}\left(\mathscr{L}\left(B_{\tau}\right)\right)$, and it is also bracketpreserving on $\mathfrak{g}_{+}$and on $\mathfrak{g}_{-}$. By using these properties we can conclude inductively that $\varphi$ is bracket-preserving on the whole $g$. In order to show (3.25), it is enough to verify it for an element of loc $(\mathrm{g})$. Let $a=[\tau(u), v]$ $\in \mathfrak{g}_{0}$, where $u, v \in \mathfrak{g}_{-1}$. In view of (3.23) and the definition of $\varphi$, we have 
$\varphi \tau([\tau(u), v])=-\varphi([\tau(v), u])=-L_{u v}=\tau_{B_{\tau}}\left(L_{v u}\right)=\tau_{B_{\tau}} \varphi([\tau(u), v])$. We can easily see (3.25) for the case $a \in \mathfrak{g}_{1}$ or $a \in \mathfrak{g}_{-1}$.

Lemma 3.11. Let $\left(U_{-1}, B\right)$ and $\left(U_{-1}^{\prime}, B^{\prime}\right)$ be two GJTS's satisfying the condition (A). Then an isomorphism $\psi$ of $\left(U_{-1}, B\right)$ onto $\left(U_{-1}^{\prime}, B^{\prime}\right)$ induces a grade-preserving isomorphism $\mathscr{L}(\psi)$ of $\mathscr{L}(B)$ onto $\mathscr{L}\left(B^{\prime}\right)$. Furthermore

$$
\mathscr{L}(\psi) \tau_{B}=\tau_{B^{\prime}} \mathscr{L}(\psi),
$$

where $\tau_{B}$ and $\tau_{B^{\prime}}$ are the grade-reversing canonical involutions of $\mathscr{L}(B)$ and $\mathscr{L}\left(B^{\prime}\right)$ respectively.

Proof. Let $\mathscr{U}=\sum_{i=-\infty}^{\infty} \mathscr{U}_{i}$ and $\mathscr{U}^{\prime}=\sum_{i=-\infty}^{\infty} \mathscr{U}_{i}^{\prime}$ be the UGLA's such that $\mathscr{U}_{-1}=U_{-1}$ and $\mathscr{U}_{-1}^{\prime}=U_{-1}^{\prime}$. $\quad \psi$ extends to an isomorphism $\hat{\psi}$ of $\mathscr{U}$ onto $\mathscr{U}^{\prime}$ (cf. 3.1). Let $\mathscr{L}_{0}(B)=\mathscr{U}_{-}+\sum_{i \geqslant 0} U_{i}$ and $\mathscr{L}_{0}\left(B^{\prime}\right)=\mathscr{U}_{-}^{\prime}+\sum_{i \geqslant 0} U_{i}^{\prime}$ be the subalgebras in (3.14). All objects in loc $\left(\mathscr{L}_{0}\left(B^{\prime}\right)\right)$ are denoted by the same notations as the corresponding ones in loc $\left(\mathscr{L}_{0}(B)\right)$ but with primes. Since $\psi$ is an isomorphism between the two GJTS's, we have $\left(\hat{\psi}\left(B_{a}\right)\right)(\psi(u), \psi(v))$ $=\psi\left(B_{a}(u, v)\right)=B_{\psi(a)}^{\prime}(\psi(u), \psi(v)), u, v \in U_{-1}$. This implies $\hat{\psi}\left(B_{a}\right)=B_{\psi(a)}^{\prime}$. Also we get $\hat{\psi}\left(L_{a b}\right)=L_{\psi(a) \psi(b)}^{\prime}, a, b \in U_{-1}$. These arguments show that $\hat{\psi}$ sends $\operatorname{loc}\left(\mathscr{L}_{0}(B)\right)$ to loc $\left(\mathscr{L}_{0}\left(B^{\prime}\right)\right)$. Noting that $\mathscr{L}_{0}(B)$ and $\mathscr{L}_{0}\left(B^{\prime}\right)$ are of type $\alpha_{0}$, we have that $\hat{\psi}$ sends $\mathscr{L}_{0}(B)$ to $\mathscr{L}_{0}\left(B^{\prime}\right)$. Therefore $\hat{\psi}$ induces a gradepreserving isomorphism $\mathscr{L}(\psi)$ of $\mathscr{L}(B)$ onto $\mathscr{L}\left(B^{\prime}\right)$ (cf. (3.15)). To see (3.26) it suffices to check it on loc $(\mathscr{L}(B))$. Let us identify $\operatorname{loc}(\mathscr{L}(B))$ with loc $\left(\mathscr{L}_{0}(B)\right)$ etc. For $L_{a b} \in U_{0}$, we have $\mathscr{L}(\psi) \tau_{B}\left(L_{a b}\right)=\mathscr{L}(\psi)\left(-L_{b a}\right)=-L_{\psi(b) \psi(a)}^{\prime}$ $=\tau_{B^{\prime}}\left(L_{\psi(a) \psi(b)}^{\prime}\right)=\tau_{B^{\prime}} \mathscr{L}(\psi)\left(L_{a b}\right)$. The remaining cases are also easily derived.

TheOREM 3.12. Let $\mathfrak{g}=\sum_{i=-\infty}^{\infty} \mathfrak{g}_{i}$ and $\mathfrak{g}^{\prime}=\sum_{i=-\infty}^{\infty} \mathfrak{g}_{i}^{\prime}$ be two real simple GLA's of type $\alpha_{0}$. Let $\tau$ and $\tau^{\prime}$ be grade-reversing involutive automorphisms of $\mathfrak{g}$ and $\mathfrak{g}^{\prime}$, respectively. Then the GJTS's $\left(\mathfrak{g}_{-1}, B_{\tau}\right)$ and $\left(\mathfrak{g}_{-1}^{\prime}, B_{\tau^{\prime}}\right)$ are isomorphic if and only if there exists a grade-preserving isomorphism $\theta$ of $\mathrm{g}$ onto $\mathfrak{g}^{\prime}$ such that

$$
\theta \tau=\tau^{\prime} \theta .
$$

Proof. As is seen from what was pointed out at the beginning of this paragraph, $\mathscr{L}\left(B_{\imath}\right)$ and $\mathscr{L}\left(B_{\tau^{\prime}}\right)$ admit the grade-reversing canonical involutions $\tau_{B_{\tau}}$ and $\tau_{B_{\tau}}$, respectively. Suppose that there exists an isomorphism $\psi$ of $\left(\mathfrak{g}_{-1}, B_{\tau}\right)$ onto $\left(\mathfrak{g}_{-1}^{\prime}, B_{\tau^{\prime}}\right)$. By Lemma 3.11 , we obtain the grade-preserving isomorphism $\mathscr{L}(\psi)$ of $\mathscr{L}\left(B_{\tau}\right)$ onto $\mathscr{L}\left(B_{\tau^{\prime}}\right)$ satisfying

$$
\mathscr{L}(\psi) \tau_{B_{\tau}}=\tau_{B_{\tau^{\prime}}} \mathscr{L}(\psi)
$$


On the other hand, by Lemma 3.10 , one can find grade-preserving isomorphisms $\varphi: \mathfrak{g} \rightarrow \mathscr{L}\left(B_{\tau}\right)$ and $\varphi^{\prime}: \mathfrak{g}^{\prime} \rightarrow \mathscr{L}\left(B_{\tau^{\prime}}\right)$ which satisfy the conditions $\varphi \tau=\tau_{B_{\tau}} \varphi$ and $\varphi^{\prime} \tau^{\prime}=\tau_{B_{\tau}} \varphi^{\prime}$. Consequently the composite map $\theta=\varphi^{\prime-1} \mathscr{L}(\psi) \varphi$ is seen to be the desired one. The converse assertion is easily seen.

3.6. We apply the results in 3.5 to the finite-dimensional case.

Definition 3.13. Let $\mathfrak{g}=\sum_{k} \mathfrak{g}_{k}$ be a real simple GLA of the first or the second kind, and $\tau$ be a grade-reversing Cartan involution of $g$. The pair $(\mathfrak{g}, \tau)$ is called an admissible pair. We say that two admissible pairs $(\mathfrak{g}, \tau)$ and $\left(\mathfrak{g}^{\prime}, \tau^{\prime}\right)$ are isomorphic, if there exists a grade-preserving isomorphism $\varphi$ of $g$ onto $g^{\prime}$ such that $\varphi \tau=\tau^{\prime} \varphi$.

We have the following classification theorem for compact GJTS's of the first or the second kind.

THEOREM 3.14. Let $\mathscr{B}$ be the set of isomorphism classes of compact real simple GJTS's of the first or the second kind, and let $\mathscr{A}$ be the set of isomorphism classes of admissible pairs. Then there exists a bijection $\Psi$ of $\mathscr{A}$ onto $\mathscr{B}$.

Proof. As was mentioned in 3.4, for a compact real GJTS of the first or the second kind, simplicity and $K$-simplicity are identical. Let $(\mathfrak{g}, \tau)$ be an admissible pair, and let $\mathfrak{g}=\sum_{l=-\nu}^{\nu} \mathfrak{g}_{i}(\nu=1,2)$. The condition $\nu=1,2$ implies that $\mathfrak{g}$ is of type $\alpha_{0}$ (cf. Lemma 2.8). Hence, by Theorem 3.5 and Lemma 3.10, the pair $\left(\mathfrak{g}_{-1}, B_{\tau}\right)$ is a simple GJTS of the $\nu$-th kind $(\nu=1,2)$. Furthermore, since $\tau$ is a Cartan involution, $\left(\mathfrak{g}_{-1}, B_{\tau}\right)$ is compact (cf. 3.4). We put

$$
\Psi([(\mathfrak{g}, \tau)])=\left[\left(\mathfrak{g}_{-1}, B_{\tau}\right)\right],
$$

where [ ] denotes an isomorphism class. From Theorem 3.12 it follows that $\Psi: \mathscr{A} \rightarrow \mathscr{B}$ is well-defined and injective. Now choose an element $\left[\left(U_{-1}, B\right)\right] \in \mathscr{B}$. Since $\left(U_{-1}, B\right)$ is simple of the $\nu$-th kind, $\mathscr{L}(B)$ is simple of the $\nu$-th kind (cf. 3.4 and Theorem 3.5). $\mathscr{L}(B)$ admits the grade-reversing canonical involution $\tau_{B}$, which is a Cartan involution by the assumption for $\left(U_{-1}, B\right)$. Consequently the pair $\left(\mathscr{L}(B), \tau_{B}\right)$ is admissible. Furthermore, for $x, y, z \in U_{-1}$ we have

$$
B(y, x, z)=B_{x}(y, z)=\left[\left[B_{x}, y\right], z\right]=\left[\left[\tau_{B}(x), y\right], z\right],
$$

which implies $B=B_{\tau_{B}}$. Therefore we get $\Psi\left(\left[\left(\mathscr{L}(B), \tau_{B}\right)\right]\right)=\left[\left(U_{-1}, B\right)\right]$. 


\section{§4. Classification of compact classical simple GJTS's}

4.1. A real GJTS $\left(U_{-1}, B\right)$ is called classical simple, if $\mathscr{L}(B)$ is classical simple. In order to classify all compact classical simple GJTS's of the first or the second kind, we have to determine the set $\mathscr{A}$ (cf. Theorem 3.14). This will be carried out by

(4.1) finding all gradations of the $\nu$-th kind $(\nu=1,2)$ in each real simple Lie algebra $g$ up to isomorphisms and by

(4.2) classifying all grade-reversing Cartan involutions for each gradation in $\mathfrak{g}$, up to conjugacy under automorphisms of the gradation.

(4.2) has been settled in Lemma 1.4; there exists a single conjugacy class of grade-reversing Cartan involutions for each gradation. (4.1) will be settled in 4.2 by using the results in 2.3. The next task is to determine $\mathfrak{g}_{-1}$ explicitly for each gradation $\left(\mathfrak{g}_{k}\right)$ and to find explicitly a grade-reversing Cartan involution. Thus we will be able to compute the GJTS's $B_{\tau}$ we are seeking (cf. (3.29)), by means of (3.16).

4.2. We refer to Takeuchi [15] for the realizations of classical real simple Lie algebras $\mathfrak{g}$ and the choices of the maximal abelian subspaces $\mathfrak{a}$, on which the root systems $\Delta$ in 2.3 are defined. We can then compute a root vector corresponding to each $\alpha \in \Delta$; by virtue of (1.23), (1.24) and

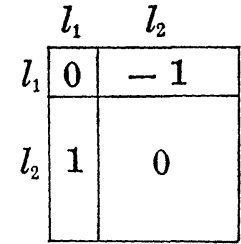

(I1) $\iota_{\iota_{1}, l_{2}}$

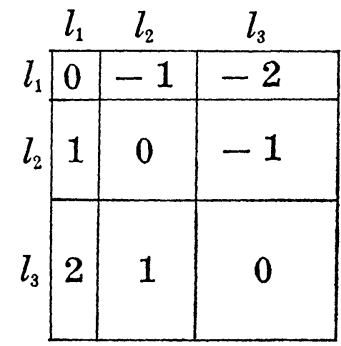

(III) $)_{l_{1}, l_{2}, l_{3}}$

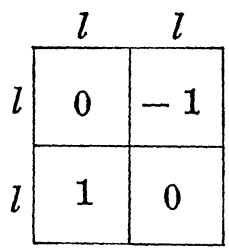

(I2)

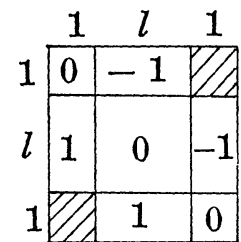

(I3)

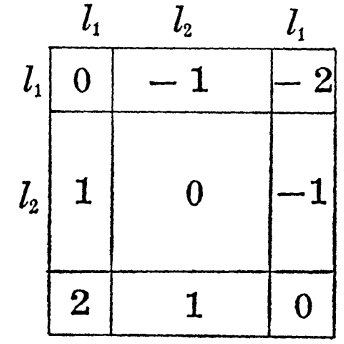

(II2) $\iota_{1}, l_{2}$

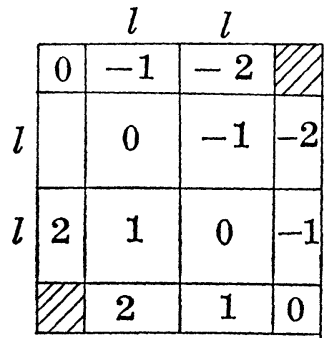

(III) 
2.3 one can find all the graded subspaces of $\mathrm{g}$. It turns out by case-bycase checking that, for classical real simple Lie algebras g, every gradation of the first or the second kind falls into one of the six types given in the page 106. For each diagram above the big square indicates an element of $\mathfrak{g}$, a matrix. The integer $k$ put in each divided portion indicates that that portion lies in the graded subspace $g_{k}$. The number beside each edge denotes the size of the portion. A shaded portion indicates that that portion does not belong to $\mathrm{g}$.

We use the following notations for the matrices: $X^{*}={ }^{t} \bar{X}$, where $X$ is a real or complex matrix. $E_{l}$ denotes the unit matrix of degree $l$. $J_{l}=\left(a_{i j}\right)$ is the $l \times l$ matrix with $a_{i j}=\delta_{i, l+1-j}$.

$$
\begin{aligned}
& \tilde{J}_{l}=\left(\begin{array}{cc}
0 & J_{l} \\
-J_{l} & 0
\end{array}\right), \quad \hat{J}_{l}=E_{l} \otimes \tilde{J}_{1}, \quad J_{l}^{\prime}=J_{l} \otimes E_{2}, \\
& K_{1}=\operatorname{diag}(\sqrt{-1},-\sqrt{-1}), \quad K_{l}=J_{l} \otimes K_{1}, \\
& A_{p, q}=\left(\begin{array}{ccc}
0 & 0 & J_{p} \\
0 & E_{q-p} & 0 \\
J_{p} & 0 & 0
\end{array}\right)(p \leqslant q), \quad A_{p, q}^{\prime}=A_{p, q} \otimes E_{2} .
\end{aligned}
$$

We also use the following notations for the vector spaces: $M_{p, q}(K)$ denotes the vector space of $p \times q$ matrices with entries in $K$, where $K=R$ or $C$.

$$
\begin{aligned}
& M_{p, q}(\boldsymbol{H})=\left\{X \in M_{2 p, 2 q}(C): \bar{X} \hat{J}_{q}=\hat{J}_{p} X\right\}, \\
& S H_{n}^{\prime}(C)=\left\{X \in \mathfrak{g l}(n, C): J_{n} X^{*} J_{n}=-X\right\} \text {, } \\
& S H_{n}^{\prime}(\boldsymbol{H})=\left\{X \in M_{n, n}(\boldsymbol{H}): J_{n}^{\prime} X^{*} J_{n}^{\prime}=-X\right\} \text {, } \\
& A l t_{n}^{\prime}(K)=\left\{X \in \operatorname{gl}(n, K): J_{n}{ }^{t} X J_{n}=-X\right\}, K=R \text { or } C \text {, } \\
& H_{n}^{\prime}(\boldsymbol{H})=\left\{X \in \mathfrak{g} \mathfrak{l}(2 n, C): \bar{X} \hat{J}_{n}=\hat{J}_{n} X, X^{*} K_{n}+K_{n} X=0\right\} \text {, } \\
& \operatorname{Sym}_{n}^{\prime}(\boldsymbol{K})=\left\{X \in \mathfrak{g} \backslash(n, K): J_{n}{ }^{t} X J_{n}=X\right\}, K=\boldsymbol{R} \text { or } \boldsymbol{C} \text {. }
\end{aligned}
$$

Table I (resp. Table II) is the list of all possible gradations of the first (resp. second) kind (up to isomorphisms) in classical real simple Lie algebras $\mathfrak{g}$ and the corresponding graded subspaces $g_{-1}$. Note that every gradation of the first or the second kind is determined by $\Pi_{1}$ (cf. 2.3).

Let $\left(g_{k}\right)$ be an arbitrary gradation in a real simple Lie algebra $\mathfrak{g}$ listed in Table I or II. It is easy to see that if we put $\tau(X)=-X^{*}$ $(X \in \mathfrak{g})$, then $\tau$ is a grade-reversing Cartan involution of the GLA $\mathfrak{g}=\sum_{k} \mathfrak{g}_{k}$. Table III (resp. Table IV) is the list of JTS's (resp. GJTS's of the second 


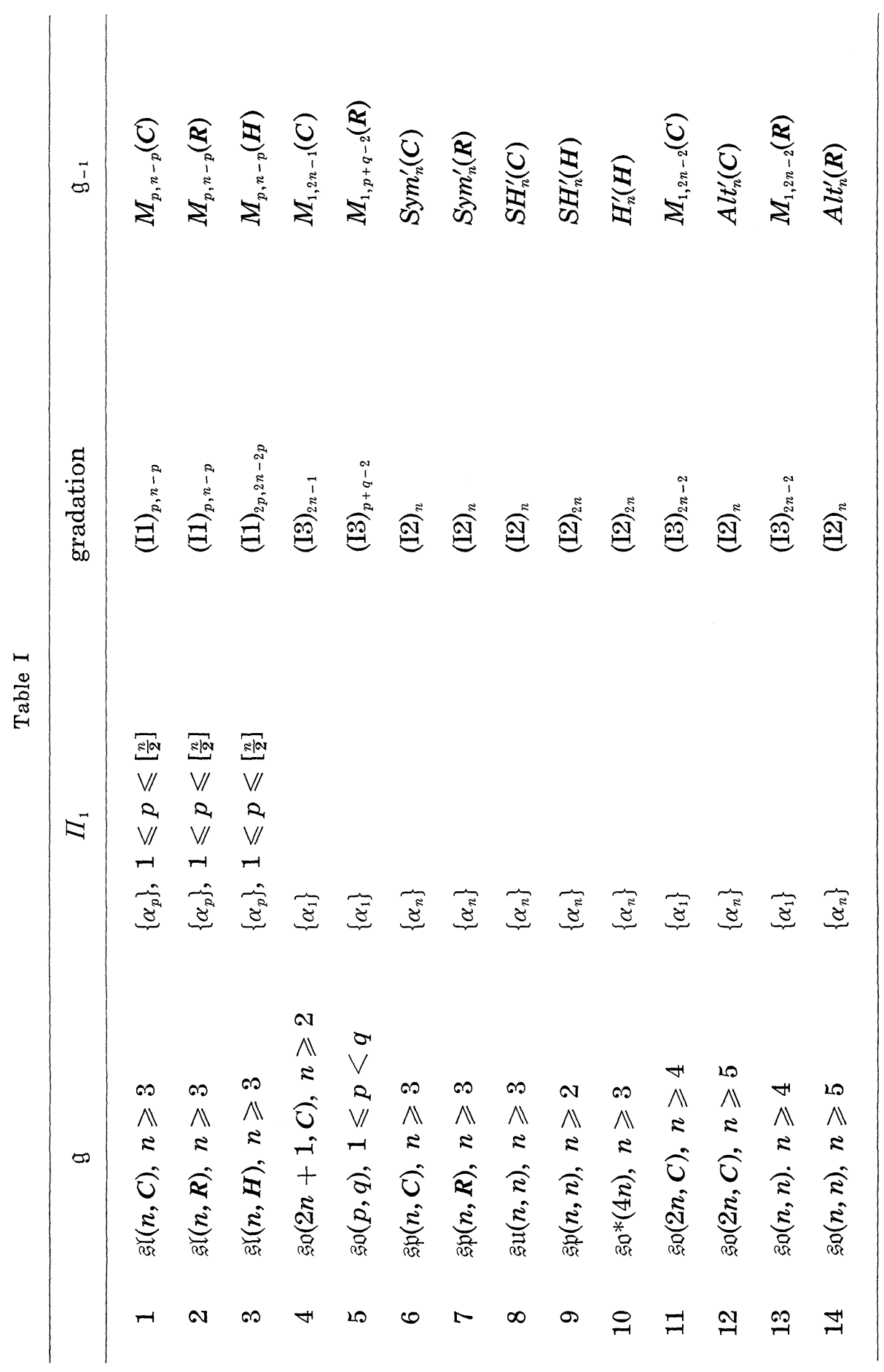




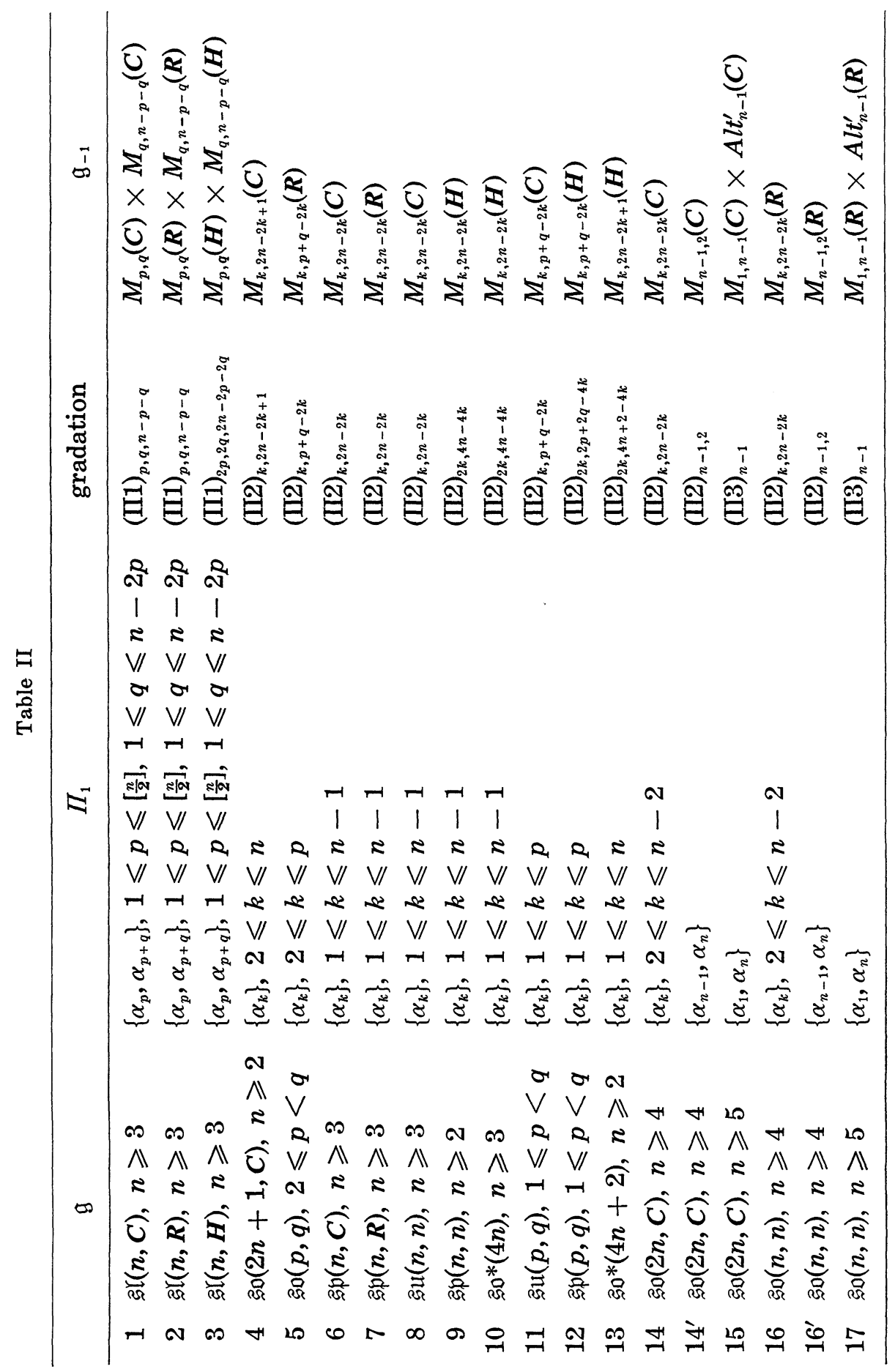


Table III

\begin{tabular}{rll}
\hline & \multicolumn{1}{c}{$\mathfrak{g}_{-1}$} & \multicolumn{1}{c}{$B_{\tau}(Y, X, Z)$} \\
\hline 1 & $M_{p, n-p}(\boldsymbol{C})$ & $Y X^{*} Z+Z X^{*} Y$ \\
2 & $M_{p, n-p}(\boldsymbol{R})$ & $Y^{t} X Z+Z^{t} X Y$ \\
3 & $M_{p, n-p}(\boldsymbol{H})$ & $Y X^{*} Z+Z X^{*} Y$ \\
4 & $M_{1,2 n-1}(\boldsymbol{C})$ & $Y X^{*} Z+Z X^{*} Y-Z J_{2 n-1}{ }^{t} Y \bar{X} J_{2 n-1}$ \\
5 & $M_{1, p+q-2}(\boldsymbol{R})$ & $Y^{t} X Z+Z^{t} X Y-Z A_{p-1, q-1}{ }^{t} Y X A_{p-1, q-1}$ \\
6 & $S y m_{n}^{\prime}(\boldsymbol{C})$ & $Y X^{*} Z+Z X^{*} Y$ \\
7 & $S y m_{n}^{\prime}(\boldsymbol{R})$ & $Y^{t} X Z+Z^{t} X Y$ \\
8 & $S H_{n}^{\prime}(\boldsymbol{C})$ & $Y X^{*} Z+Z X^{*} Y$ \\
9 & $S H_{n}^{\prime}(\boldsymbol{H})$ & $Y X^{*} Z+Z X^{*} Y$ \\
10 & $H_{n}^{\prime}(\boldsymbol{H})$ & $Y X^{*} Z+Z X^{*} Y$ \\
11 & $M_{1,2 n-2}(\boldsymbol{C})$ & $Y X^{*} Z+Z X^{*} Y-Z J_{2 n-2}{ }^{t} Y \bar{X} J_{2 n-2}$ \\
12 & $A l t_{n}^{\prime}(\boldsymbol{C})$ & $Y X^{*} Z+Z X^{*} Y$ \\
13 & $M_{1,2 n-2}(\boldsymbol{R})$ & $Y^{t} X Z+Z^{t} X Y-Z J_{2 n-2}{ }^{t} Y X J_{2 n-2}$ \\
14 & $A l t_{n}^{\prime}(\boldsymbol{R})$ & $Y^{t} X Z+Z^{t} X Y$
\end{tabular}

Table IV

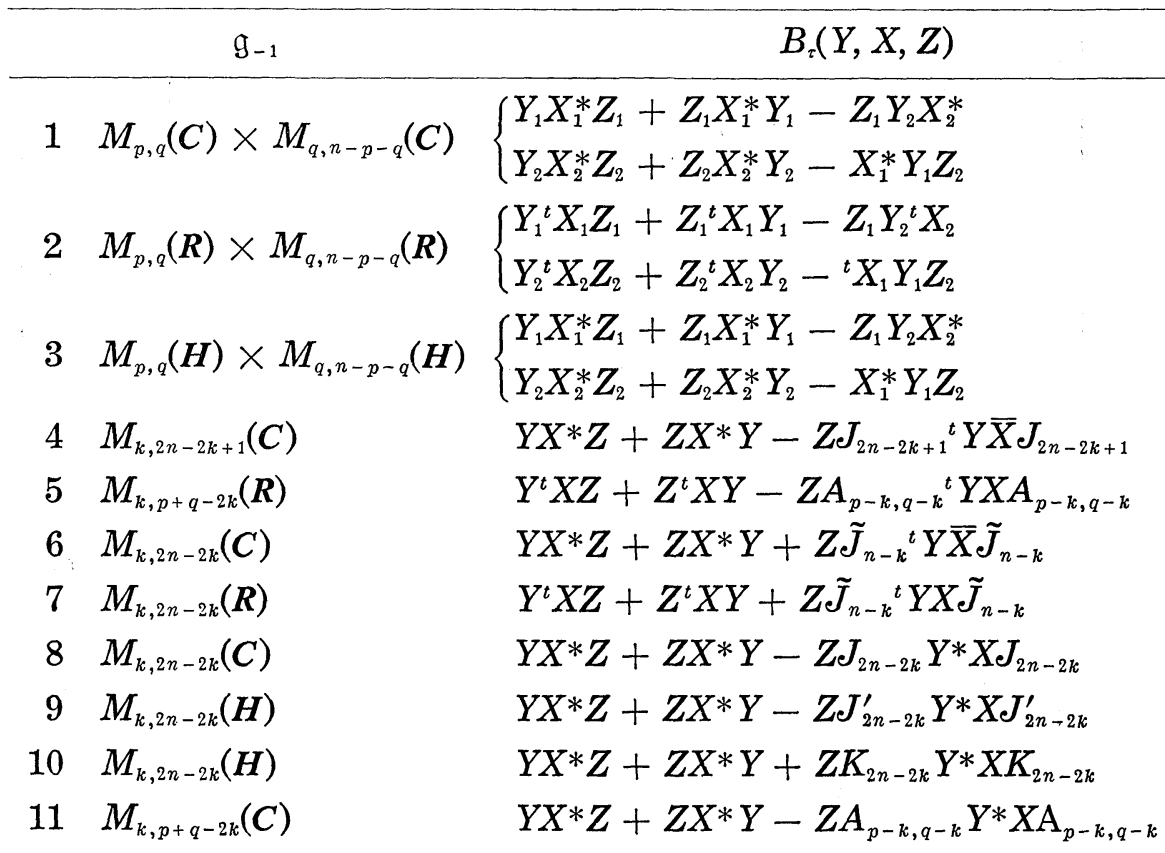




\begin{tabular}{|c|c|}
\hline $2 \quad M_{k, p+q-2 k}(\boldsymbol{H})$ & $Y X^{*} Z+Z X^{*} Y-Z A_{p-k, q-k}^{\prime} Y^{*} X A_{p-k, q-k}^{\prime}$ \\
\hline$M_{k, 2 n-2 k+1}(\boldsymbol{H})$ & $Y X^{*} Z+Z X^{*} Y+Z K_{2 n-2 k+1} Y^{*} X K_{2 n-2 k+1}$ \\
\hline$M_{k, 2 n-2 k}(C)$ & $Y X^{*} Z+Z X^{*} Y-Z J_{2 n-2 k}^{t} Y \bar{X} J_{2 n-2 k}$ \\
\hline$M_{1, n-1}(C) \times A l t_{n-1}^{\prime}(C)$ & $\left\{\begin{array}{l}Y_{1} X_{1}^{*} Z_{1}+Z_{1} X_{1}^{*} Y_{1}-Z_{1} Y_{2} X_{2}^{*} \\
Y_{2} X_{2}^{*} Z_{2}+Z_{2} X_{2}^{*} Y_{2}-X_{1}^{*} Y_{1} Z_{2}-Z_{2} J_{n-1}{ }^{t} Y_{1} \bar{X}_{1} J_{n-1}\end{array}\right.$ \\
\hline$M_{k, 2 n-2 k}(R)$ & $Y^{t} X Z+Z^{t} X Y-Z J_{2 n-2 k}^{t} Y X J_{2 n-2 k}$ \\
\hline$M_{1, n-1}(R) \times A l t_{n-1}^{\prime}(R)$ & $\left\{\begin{array}{l}Y_{1}^{t} X_{1} Z_{1}+Z_{1}^{t} X_{1} Y_{1}-Z_{1} Y_{2}^{t} X_{2} \\
Y_{2}^{t} X_{2} Z_{2}+Z_{2}^{t} X_{2} Y_{2}-{ }^{t} X_{1} Y_{1} Z_{2}-Z_{2} J_{n-1}{ }^{t} Y_{1} X_{1} J_{n-1}\end{array}\right.$ \\
\hline
\end{tabular}

kind) $\left(\mathfrak{g}_{-1}, B_{\tau}\right)$ which are obtained from the gradations given in Table I (resp. Table II). In Table IV, if $g_{-1}$ is a direct product of two vector spaces $V_{1}$

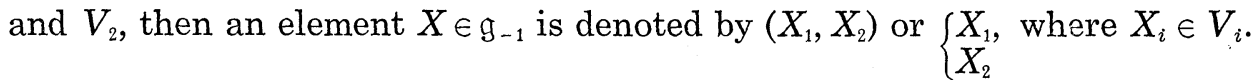

From Table III we have the following

TheORem 4.1. Compact classical real simple JTS's $\left(U_{-1}, B\right)$ are classified (up to isomorphisms) as follows:

(1) $U_{-1}=M_{p, q}(K), K=R, C, H ; p \leqslant q$,

$$
B(Y, X, Z)=Y X^{*} Z+Z X^{*} Y \text {. }
$$

(2) $U_{-1}=\operatorname{Sym}_{n}^{\prime}(K), K=R, C ; n \geqslant 3$,

$$
B(Y, X, Z)=Y X^{*} Z+Z X^{*} Y \text {. }
$$

(3) $U_{-1}=A l t_{n}^{\prime}(K), K=R, C ; n \geqslant 5$,

$$
B(Y, X, Z)=Y X^{*} Z+Z X^{*} Y \text {. }
$$

(4) $U_{-1}=S H_{n}^{\prime}(K), K=C, H ; n \geqslant 3$ for $K=C, n \geqslant 2$ for $K=H$,

$$
B(Y, X, Z)=Y X^{*} Z+Z X^{*} Y \text {. }
$$

(5) $U_{-1}=H_{n}^{\prime}(\boldsymbol{H}), n \geqslant 3$,

$$
B(Y, X, Z)=Y X^{*} Z+Z X^{*} Y .
$$

(6) $U_{-1}=M_{1, n}(C), n \geqslant 3, n \neq 4$,

$$
B(Y, X, Z)=Y X^{*} Z+Z X^{*} Y-Z J_{n}^{t} Y \bar{X} J_{n} .
$$

$$
\begin{aligned}
& U_{-1}=M_{1, p+q}(R), 0 \leqslant p<q \text { or } 3 \leqslant p=q, \\
& B(Y, X, Z)=Y^{t} X Z+Z^{t} X Y-Z A_{p, q}{ }^{t} Y X A_{p, q} .
\end{aligned}
$$

It is easy to see that the above result is essentially the same as the 
one in Loos [10]. From Table IV we have

Theorem 4.2. Compact classical real simple GJTS's $\left(U_{-1}, B\right)$ of the second kind are classified (up to isomorphisms) as follows:

$$
\begin{aligned}
& U_{-1}=M_{p, q}(K) \times M_{q, r}(K), K=R, C, H, \\
& 1 \leqslant p \leqslant\left[\frac{n}{2}\right], \quad p \leqslant r, \quad p+q+r=n, \\
& B(Y, X, Z)=\left(\begin{array}{rl}
Y_{1} X_{1}^{*} Z_{1}+ & Z_{1} X_{1}^{*} Y_{1}-Z_{1} Y_{2} X_{2}^{*}, \\
& \left.Y_{2} X_{2}^{*} Z_{2}+Z_{2} X_{2}^{*} Y_{2}-X_{1}^{*} Y_{1} Z_{2}\right),
\end{array}\right.
\end{aligned}
$$

where

$$
X=\left(X_{1}, X_{2}\right), \quad Y=\left(Y_{1}, Y_{2}\right), \quad Z=\left(Z_{1}, Z_{2}\right) .
$$

(2) $U_{-1}=M_{k, p+q-2 k}(K), K=R, C$,

$$
\begin{aligned}
& 2 \leqslant k \leqslant p<q \text { or } 2 \leqslant k<p=q(\geqslant 4) \text { for } \boldsymbol{K}=\boldsymbol{R}, \\
& 1 \leqslant k \leqslant p<q \text { or } 1 \leqslant k<p=q(\geqslant 4) \text { for } \boldsymbol{K}=\boldsymbol{C}, \\
& B(Y, X, Z)=Y X^{*} Z+Z X^{*} Y-Z A_{p-k, q-k} Y^{*} X A_{p-k, q-k} .
\end{aligned}
$$

(3) $U_{-1}=M_{k, p+q-2 k}(H)$,

$$
\begin{gathered}
1 \leqslant k \leqslant p<q \text { or } 1 \leqslant k<p=q \\
B(Y, X, Z)=Y X^{*} Z+Z X^{*} Y-Z A_{p-k, q-k}^{\prime} Y^{*} X A_{p-k, q-k}^{\prime} .
\end{gathered}
$$

(4) $U_{-1}=M_{k, m-2 k}(C)$,

$$
\begin{array}{cc}
2 \leqslant k \leqslant n & \text { for } m=2 n+1, \\
2 \leqslant k \leqslant n-1 \quad \text { for } m=2 n(n \geqslant 4), \\
B(Y, X, Z)=Y X^{*} Z+Z X^{*} Y-Z J_{m-2 k}{ }^{t} Y \bar{X} J_{m-2 k} .
\end{array}
$$

(5) $U_{-1}=M_{k, m-2 k}(\boldsymbol{H})$,

$$
\begin{gathered}
1 \leqslant k \leqslant n \quad \quad \text { for } m=2 n+1(n \geqslant 2), \\
1 \leqslant k \leqslant n-1 \quad \text { for } m=2 n(n \geqslant 3), \\
B(Y, X, Z)=Y X^{*} Z+Z X^{*} Y+Z K_{m-2 k} Y^{*} X K_{m-2 k} .
\end{gathered}
$$

(6) $U_{-1}=M_{k, 2 n-2 k}(K), K=R, C$,

$$
1 \leqslant k \leqslant n-1(n \geqslant 3),
$$

$$
B(Y, X, Z)=Y X^{*} Z+Z X^{*} Y+Z \tilde{J}_{n-k}{ }^{t} Y \bar{X} \tilde{J}_{n-k} .
$$

(7) $U_{-1}=M_{1, n-1}(K) \times A l t_{n-1}^{\prime}(K), K=R, C ; n \geqslant 5$, 


$$
\begin{aligned}
B(Y, X, Z)= & \left(Y_{1} X_{1}^{*} Z_{1}+Z_{1} X_{1}^{*} Y_{1}-Z_{1} Y_{2} X_{2}^{*},\right. \\
& \left.Y_{2} X_{2}^{*} Z_{2}+Z_{2} X_{2}^{*} Y-X_{1}^{*} Y_{1} Z_{2}-Z_{2} J_{n-1}{ }^{t} Y_{1} \bar{X}_{\mathrm{i}} J_{n-1}\right),
\end{aligned}
$$

where

$$
X=\left(X_{1}, X_{2}\right), \quad Y=\left(Y_{1}, Y_{2}\right), \quad Z=\left(Z_{1}, Z_{2}\right) .
$$

\section{$\S 5$. $\varepsilon$-modifications of compact simple GJTS's}

We will give here a method of constructing noncompact simple GJTS's, starting from compact simple GJTS's. Let $\left(U_{-1}, B\right)$ be a compact real simple GJTS of the $\nu$-th kind $(\nu=1,2)$. Then we have the admissible pair $\left(\mathscr{L}(B), \tau_{B}\right)$ (cf. the proof of Theorem 3.14). $\mathscr{L}(B)$ is a simple GLA of type $\alpha_{0}$ of the $\nu$-th kind $(\nu=1,2)$. For brevity we put $g=\mathscr{L}(B)$ and $\tau=\tau_{B}$. Let $\mathfrak{g}=\sum_{k=-\nu}^{\nu} \mathfrak{g}_{k}$ and let $E$ be its characteristic element. Note that $\mathrm{g}_{-1}=U_{-1}$. $\tau$ is a grade-reversing Cartan involution of $\mathrm{g}$. We choose a maximal abelian subspace a containing $E$ satisfying (1.1). Let $\Delta$ be the root system of $g$ with respect to $a$. Now consider a signature $\varepsilon$ of roots in $\Delta$ in the sense of Oshima-Sekiguchi [12], and let $\tau_{\varepsilon}$ be the $\varepsilon$-modification of $\tau$. $\tau_{\varepsilon}$ is also an involutive automorphism of $\mathfrak{g}$.

Proposition 5.1. Let

$$
B_{\varepsilon}(Y, X, Z)=\left[\left[\tau_{\varepsilon}(X), Y\right], Z\right] \quad X, Y, Z \in U_{-1} .
$$

Then $\left(U_{-1}, B_{\varepsilon}\right)$ is a noncompact simple GJTS of the $\nu$-th kind $(\nu=1,2)$.

Proof. In view of the definition of $\tau_{\varepsilon}$ [12], it follows that $\tau_{\varepsilon}$ coincides with $\tau$ on $a$. Hence we have $\tau_{\varepsilon}(E)=-E$ and consequently $\tau_{\varepsilon}$ is a grade-reversing involutive automorphism of $\mathfrak{g}$. By Theorem 3.5, $\left(U_{-1}, B_{\varepsilon}\right)$ is a $K$-simple GJTS and hence it is simple (cf. 3.4). Consequently $\left(U_{-1}, B_{s}\right)$ satisfies the condition (A). The GLA $\mathscr{L}\left(B_{\varepsilon}\right)$ admits the grade-reversing canonical involution $\tau_{B_{s}}$ (cf. Proposition 3.8). By Lemma 3.10, there exists a grade-preserving isomorphism $\varphi$ of $\mathfrak{g}$ onto $\mathscr{L}\left(B_{\varepsilon}\right)$ such that $\varphi \tau_{\varepsilon}=$ $\tau_{B_{\varepsilon}} \varphi$. Since $\tau_{\varepsilon}$ is not a Cartan involution, $\tau_{B_{\varepsilon}}$ is not either. Therefore $B_{\varepsilon}$ is not compact [1].

We say that $\left(U_{-1}, B_{\varepsilon}\right)$ is an $\varepsilon$-modification of $\left(U_{-1}, B\right)$.

Remark 5.2. Let $\mathfrak{g}=\sum_{k} \mathrm{~g}_{k}$ be a simple GLA of type $\alpha_{0}$ of the $\nu$-th kind $(\nu=1,2)$. Let $\tau$ be a grade-reversing Cartan involution of $\mathfrak{g}$ and $\tau_{\varepsilon}$ be an $\varepsilon$-modification of $\tau$. The proof of the above theorem shows that $B_{\varepsilon}$ in (5.1) is a noncompact simple GJTS of the $\nu$-th kind $(\nu=1,2)$. 
EXAMPle 5.3. Let us consider the gradation (II2) $)_{2,4}$ of $\mathfrak{g}=\mathfrak{g} \mathfrak{u}(4,4)$ (cf. Table II). In this case $\mathfrak{g}_{-1}=M_{2,4}(C)$. A grade-reversing Cartan involution is given by $\tau$ such that $\tau(X)=-X^{*}, X \in \mathfrak{s} \mathfrak{u}(4,4)$. The corresponding compact simple GJTS is found in Table IV. Let $\Pi=\left\{\alpha_{1}, \alpha_{2}, \alpha_{3}, \alpha_{4}\right\}$ be a fundamental system for $\Delta$ compatible with the gradation. We define a signature $\varepsilon$ by $\varepsilon\left(\alpha_{1}\right)=-1$ and $\varepsilon\left(\alpha_{i}\right)=1$ for $i=2,3,4$ ([12]). By easy computations we can verify that $\tau_{\varepsilon}(X)=A \tau(X) A$ for $X \in \mathfrak{I} \mathfrak{u}(4,4)$, where $A=\operatorname{diag}\left(-1, E_{6},-1\right)$. By direct computations we see for $X, Y, Z \in$ $M_{2,4}(C)$

$$
B_{\varepsilon}(Y, X, Z)=Y X^{*} I_{1,1} Z+Z X^{*} I_{1,1} Y-Z J_{4} Y^{*} I_{1,1} X J_{4},
$$

where $I_{1,1}=\operatorname{diag}(-1,1)$. The above $B_{\varepsilon}$ provides an example of noncompact simple GJTS's.

\section{BIBLIOGRAPHY}

[1] H. Asano and S. Kaneyuki, On compact generalized Jordan triple systems, Tokyo J. Math., 11 (1988), 105-118.

[2] N. Bourbaki, Groupes et Algèbres de Lie, Chap. II, Hermann, Paris, 1971.

[ 3 ] J. H. Cheng, Graded Lie algebras of the second kind, Trans. Amer. Math. Soc., 302 (1987), 467-488.

[4] S. Kobayashi and T. Nagano, On filtered Lie algebras and geometric structures I, J. Math. Mech., 13 (1964), 875-908.

[5] V. G. Kac, Some remarks on nilpotent orbits, J. Algebra, 64 (1980), 190-213.

[6] I. L. Kantor, Graded Lie algebras, Trudy Sem. Vekt. Tenz. Anal., 15 (1970), 227-266.

[ 7 ] - Some generalizations of Jordan algebras, Trudy Sem. Vekt. Tenz. Anal., 16 (1972), 407-499.

[ 8 ] - Models of exceptional Lie algebras, Soviet Math. Dokl., 14 (1973), 254-258.

[9] M. Koecher, An Elementary Approach to Bounded Symmetric Domains, Lect. Notes, Rice Univ., Houston, 1969.

[10] O. Loos, Bounded Symmetric Domains and Jordan Pairs, Math. Lect., Univ. Calif., Irvine, 1977.

[11] E. Neher, Cartan-Involutionen von halbeinfachen reellen Jordan-Tripelsystemen, Math. Z., 169 (1979), 271-292.

[12] T. Oshima and J. Sekiguchi, Eigenspaces of invariant differential operators on an affine symmetric spaces, Invent. Math., 57 (1980), 1-81.

[13] I. Satake, On representations and compactifications of symmetric Riemannian spaces, Ann. of Math., 71 (1960), 77-110.

[14] — Algebraic Structures of Symmetric Domains, Iwanami Shoten, Tokyo and Princeton Univ. Press, Princeton, 1980.

[15] M. Takeuchi, Cell decompositions and Morse equalities on certain symmetric spaces, 
J. Fac. Sci. Univ. Tokyo, 12 (1965), 81-192.

[16] N. Tanaka, On non-degenerate real hypersurfaces, graded Lie algebras and Cartan connections, Japan. J. Math., 2 (1976), 131-190.

[17] — On the equivalence problems associated with simple graded Lie algebras, Hokkaido Math. J., 8 (1979), 23-84.

[18] - On affine symmetric spaces and the automorphism groups of product manifolds, Hokkaido Math. J., 14 (1985), 277-351.

[19] K. Yamaguti, On the metasymplectic geometry and triple systems, Kokyuroku, 308 (1977), 55-92, RIMS, Kyoto Univ. (in Japanese).

Soji Kaneyuki

Department of Mathematics

Sophia University

Kioicho, Tokyo 102,

Hiroshi Asano

Department of Mathematics

Yokohama City University

Seto, Yokohama 236 